\title{
On the Transferability of CHARMM36m Protein Force Field with LJ-PME: Hydrogen Bonding Dynamics under Elevated Pressures
}

\author{
You $\mathrm{Xu},{ }^{1,2,3}$ Jing Huang ${ }^{1,2,3} *$
}

1. Key Laboratory of Structural Biology of Zhejiang Province, School of Life Sciences, Westlake University, 18 Shilongshan Road, Hangzhou, Zhejiang 310024, China.

2. Westlake Laboratory of Life Sciences and Biomedicine, 18 Shilongshan Road, Hangzhou, Zhejiang 310024, China.

3. Institute of Biology, Westlake Institute for Advanced Study, 18 Shilongshan Road, Hangzhou, Zhejiang 310024, China.

*Corresponding author: Jing Huang, huangjing@ westlake.edu.cn 


\begin{abstract}
The pressure-temperature phase diagram is significant to understanding the physics of biomolecules. Compared to various studies on temperature effects, researches on the dependence of protein dynamics on pressure are rather limited. Molecular dynamics simulation with finetuned force fields (FFs) is a powerful tool to explore the influence of thermodynamic condition on proteins. In this study we evaluated the transferability of CHARMM $36 \mathrm{~m}(\mathrm{C} 36 \mathrm{~m})$ protein force field in varied pressures using ubiquitin as a model protein compared with NMR data. The pressure dependences of $J$ couplings for hydrogen bonds and order parameters for internal motion were in good agreement with experiment. We demonstrate that C36m FF combining with the LJPME method is suitable for simulations in a wide range of temperature and pressure. Although the structures of ubiquitin kept stable up to 2500 bar, we identified different mobility and stability in terms of hydrogen bonds and conformation, which is helpful to understand pressure effects on ubiquitin. Herein the application of $\mathrm{C} 36 \mathrm{~m}$ is expected for more proteins in the future to better understand the pressure denaturation.
\end{abstract}

Keywords: ubiquitin, molecular simulation, CHARMM36m, force field, LJ-PME 


\section{Introduction}

The pressure-temperature $(p-T)$ phase diagram indicates the thermodynamic range in which the protein structure keeps native or denatured, thus is very informative in protein engineering. While the temperature influence on protein conformational dynamics has been extensively investigated, studies on the protein structure and function at high pressures are relatively rare. Recently the protein properties concerned high ambient pressure in bio-engineering is boosted, such as enzyme design, food industry and low temperature sterilization. ${ }^{1-5}$

Molecular simulations have emerged as important tools for unrevealing the structure-dynamicsfunction relationship of proteins. Recently simulations have been applied to not only folded proteins but also intrinsically disordered proteins, as well as proteins under complicated but functionally important environments such as multi-component membranes ${ }^{6,7}$, phase separated states $^{8,9}$, and crowded environment in cells ${ }^{10,11}$. The quality of these simulations depends critically on their underlying models, typically the empirical force fields. Protein force fields have been continuously improved; however, the refinement is usually performed under ambient conditions, so that their transferability towards different simulation conditions needs to be scrutinized carefully.

Recent advances in high pressure instrumentation as well as in the method to collect samples from the extreme environments at ocean bottom make it possible to study how the enzymes in piezophilic microbes function. The fluorescence measurements show that pressure modifies the catalysis constant by affecting the thermodynamic properties thereby was supposed to change the enzyme turnover in engineering. ${ }^{12}$ Small angle X-ray and neutron scattering experiments show that the high-pressure stability of calmodulin arises from the reduced void volume inside the protein meanwhile the internal fluctuation is enhanced. ${ }^{13}$ The transition states from coiled to folded ubiquitin in declining hydrostatic pressure were captured by nuclear magnetic resonance (NMR) measurements, so the folding pathway was suggested. ${ }^{14}$ Indeed the volume change is the key point to capture and evaluate the protein unfolding states. ${ }^{15}$ Such study will deepen our understanding of how life origins and molecular dynamics simulations have been performed at these very high pressures. ${ }^{16,17}$ Unlike temperature, which has a definitely narrow window for protein function, the hydrostatic pressure causing protein denaturation is varied and such unfolding is usually reversible. ${ }^{15,17}$ However, whether current protein force fields, which are developed and tested at $1 \mathrm{~atm}$, can be used at high pressure such as 1000 or 2000 bar remains an open question.

NMR measurements provide valuable, ensemble averaged information on protein conformations, and therefore have long been used to benchmark protein force fields. ${ }^{18-21}$ In particular, throughspace scalar coupling ${ }^{\mathrm{h} 3} \mathrm{~J}_{\mathrm{NC}}$, detects the strength of protein backbone hydrogen bond $\mathrm{N}-\mathrm{H} \cdots \mathrm{O}=\mathrm{C}$ between two residues ${ }^{22,23}$ and provides information for both the local $\mathrm{H}$-bond interaction pattern and the global protein conformational dynamics. In 2010 Lange et al compared 10 different protein FFs using the scalar coupling across hydrogen bonds ${ }^{\mathrm{h} 3} \mathrm{~J}_{\mathrm{NC}}$, and residual dipolar couplings 
(RDCs) of ubiquitin and G protein B3 domain. ${ }^{21}$ The ${ }^{\mathrm{h} 3} J_{\mathrm{NC}}$, couplings have also been used in the validation of recent force field development such as CHARMM $36 \mathrm{~m}^{24}$ and Amber ff99SB-disp ${ }^{25}$. NMR relaxation is another frequently used measurement to detect the dynamics of residue side chains, as employed frequently in ubiquitin system. ${ }^{26,}{ }^{27}$ Such NMR data can be compared with the calculated axis order parameter that is affected by the side-chain flexibility and free volume. ${ }^{28}$ A recent NMR study has collected the ${ }^{\mathrm{h} 3}{ }_{\mathrm{NC}}$, data for ubiquitin to systematically investigate the protein conformational change in response to varied ambient pressure from 1 to $2500 \mathrm{bar},{ }^{29}$ which provides new data to validate force fields.

LJ-PME is a recent advance in simulation method involving the better treatment of non-bonded Lennard-Jones (LJ) interactions, or more precisely the dispersion (C6) term in LJ potentials. Usually the pairwise LJ interactions are evaluated only within a truncated cutoff radius, for example $9 \AA$ or $12 \AA$. Although decaying with $r^{-6}$, the dispersion term is always negative so that the net sum beyond cutoff radius can be accumulated to an unneglectable value, which is important in highly anisotropic membrane protein systems as well as ligand-protein binding free energy calculations. ${ }^{30}$ LJ-PME employs particle mesh Ewald (PME) algorithm to improve the accuracy of dispersion terms as it has been widely used in electrostatic calculations. It uses Lorentz-Berthelot (LB) combination rules in real space, but the geometric mean in reciprocal space to allow the factorization for the interaction parameters of $\mathrm{C} 6$ terms. ${ }^{31}$ For common force fields, such as AMBER and CHARMM which employ LB rules for LJ energy calculation, this treatment causes extra computational work while still leaves error in reciprocal space systematically. Wennberg et al later showed that subtracting an exact term in direct space the errors from reciprocal space can be canceled out, thereby improving the accuracy. ${ }^{32}$ With respect to CHARMM force fields, the advantage on calculating long-range dispersion force of LJ-PME in simulating alkane systems has recently been illustrated. ${ }^{33}$ However, the performance of LJ-PME with CHARMM protein force field under varied thermodynamic conditions requires validation.

In this work we studied the transferability of the most recent CHARMM36m protein force field (C36m) using NMR experimental data, especially the hydrogen bond $J$ coupling data. We established that the C36m can be readily used together with LJ-PME for protein simulations. Ubiquitin was taken as the model in the MD simulations for its superior thermal stability. We compared the computational $J$ couplings and order parameters with experimental measurements under different temperature and pressure conditions. Furthermore we elaborately analyzed the hydrogen bond features of ubiquitin at elevated pressure up to 2500 bar. The study ends with a brief conclusion on the performance of $\mathrm{C} 36 \mathrm{~m}$ in thermodynamic transferability. 


\section{Methods}

\section{MD simulations}

The ubiquitin structure (PDB ID: $1 \mathrm{UBQ}^{34}, 1.8 \AA$ ) was used as the model system in different thermodynamic conditions. All the titratable residues were treated as ionized and histidine 68 was protonated on $\delta$-N. It was solvated in a TIP3P water ${ }^{35}$ box with $58.5 \AA$ as the cubic dimension. The $\mathrm{N}$ - and C-terminal were capped by standard ammonium and carbonate forms respectively and no counter-ions and salt concentration were added. The system totally has 20290 atoms. Other proteins which were investigated for validation of LJ-PME simulations include cold-shock protein A (1MJC), protein G B1 domain (2QMT, $1.1 \AA$ ), apo-calmodulin (1QX5), and intestinal fatty acid binding protein (1IFC). The setup and pre-equilibrium of these systems were done following the same procedure in a previous study ${ }^{20}$.

Periodic boundary conditions were used and the particle mesh Ewald ${ }^{36}$ were applied on both electrostatic and Lennard-Jones (LJ-PME) interactions, with $9 \AA$ as the real space cutoff and $10^{-4}$ as the error tolerance. The simulations were performed in isothermal-isobaric ensemble $(N p T)$ by employing Andersen thermostat and Monte Carlo barostat. For 1UBQ the pressure and temperature conditions were designed according to the NMR experimental study ${ }^{29}$ with a few more simulations added to make the analysis more systematic (Table 1). Simulations of all other proteins were carried out only at room condition ( $300 \mathrm{~K}$ and 1 bar). $\mathrm{C} 36 \mathrm{~m}^{24,}{ }^{37} \mathrm{FF}$ and the CHARMM modified TIP3P water model were applied. With the constraint on hydrogen involved bonds, a time step of $2 \mathrm{fs}$ was used in velocity Verlet integrator. Each system was run $1.2 \mu \mathrm{s}$ using OpenMM $7^{38}$ with snapshot saving in every 100 ps.

\section{Analysis}

The last $1 \mu$ s from each $1.2 \mu$ s trajectory including 10000 snapshots was accounted in analysis using CHARMM ${ }^{39}$. The strength of hydrogen bond quantified using the scalar coupling between the backbone $\mathrm{N}$ and $\mathrm{C}$ atoms was considered as the fingerprint to identify the structural stability. The scalar coupling is calculated using the following formula as described previously, ${ }^{40}$

$$
{ }^{\mathrm{h} 3} J_{\mathrm{NC} \prime}=\left\langle(-357 \mathrm{~Hz}) \exp \left(-3.2 r_{\mathrm{HO}}\right) \cos ^{2} \theta\right\rangle
$$

where $r_{\mathrm{HO}}$ is the distance between the hydrogen and the acceptor oxygen atom, $\theta$ is the $\mathrm{H} \cdots \mathrm{O}=\mathrm{C}$ angle, and angular bracket stands for ensemble average over the MD trajectories.

The global intramolecular hydrogen bonds in ubiquitin were identified during MD simulations considering the criteria that $r_{\mathrm{HO}} \leq 2.4 \AA$ and $\theta \geq 100^{\circ}$. For a residue which has multiple equivalent donors or acceptors involving in the interaction during the same lifetime span, these $\mathrm{H}$-bond events were degenerated to be same one. The percentage of degenerated dwell time over whole MD trajectory is called effective occupancy, and it was calculated for all H-bonds detected in simulations. The following residues have equivalent donors or acceptors: Asn (HD21, HD22), Gln (HE21, HE22), Lys (HZ1, HZ2, HZ3), Arg (HE, HH11, HH12, HH21, HH22), N-terminal (HT1, HT2, HT3), Asp (OD1, OD2), Glu (OE1, OE2), and C-terminal (OT1, OT2). The effective occupancy of water bridged hydrogen bonds was also considered, where a water molecule bonds 
two residues at the same time. Similar to H-bonds, the effective hydrophobic contact was counted for the distance between the mass centers of two hydrophobic side chains. The cutoff was $3.5 \AA$ between any pair of mass centers and no direction criterion was set.

The order parameter $S^{2}$ which can be directly related to the NMR relaxation data was calculated using the trace of tensor and taking the ensemble average, as described before, ${ }^{28}$

$$
S^{2}=\frac{3}{2} \operatorname{tr}\langle\boldsymbol{\Phi}\rangle^{2}-\frac{1}{2}(\operatorname{tr}\langle\boldsymbol{\Phi}\rangle)^{2}
$$

where $\boldsymbol{\Phi}$ is a $3 \times 3$ tensor of a unit vector along $\mathrm{N}-\mathrm{H}$ axis of backbone amide or C-C axis of side chain methyl group for each specified residue. In practice, the vectors were computed for each snapshot and averaged over the whole trajectory as the ensemble average. 


\section{Results}

\section{Performance of $\mathrm{C36m}$ in varied pressures}

\section{The LJ-PME was feasible with C36m}

As all ubiquitin simulations presented in this study were carried out using the LJ-PME method with OpenMM, we first performed some validation studies. A box of 1890 TIP3P water molecules were simulated in $N p T$ ensemble using both switching function and LJ-PME to model the Lennard-Jones interactions. The density was calculated as the function of pressure (from 1 to 2500 bar) under four different temperatures. As shown in Figure S1, the results of LJ-PME using $9 \AA$ as cutoff were equivalent to those using $30 \AA$ especially when the pressure is lower than 1000 bar. In contrast, the densities calculated using $12 \AA$ as cutoff were systematically smaller, because the long range dispersion interaction is not fully accounted. The difference is not significant for water system since electrostatic is the dominant interaction here. In contrast, we recently showed that simulation results of alkanes are more influenced by the treatment of $\mathrm{LJ}$ interactions, where with LJ-PME the experimental results were better reproduced. ${ }^{33}$

We also validated the ability of the $\mathrm{C} 36 \mathrm{~m}$ protein force fields in reproducing protein structures and dynamics in combination of the LJ-PME method, by comparing the NMR ${ }^{\mathrm{h} 3} J_{\mathrm{NC}}$ couplings for a set of five proteins. These data were used to validate the CHARMM36 and the CHARMM36m protein force fields with MD simulations run using $12-\AA$ cutoff for vdW interactions. As shown in 
Table 2, very similar correlations between calculated and experimental ${ }^{\mathrm{h} 3} J_{\mathrm{NC}}$ couplings were achieved with the cutoff method and the LJ-PME method for treating the vdW interactions. This is not surprising as for solvated protein systems the electrostatic interactions still dominate the total interaction energy. The C36m FF that was developed using cutoff method can be directly combined with the LJ-PME method to simulate protein systems.

\section{Structural flexibility of ubiquitin was partially decreased}

During the $1.2 \mu \mathrm{s}$ simulations of ubiquitin under different pressures and temperatures, all systems reached the equilibrium after first several nanoseconds, and ubiquitin kept in expected stability in varied $p-T$ conditions (Figure S2). The global root mean square deviation (RMSD) of the coordinate for non-hydrogen atoms was evaluated compared to the original 1UBQ conformation. The RMSDs were all below $2 \AA$ and there was no obvious tendency of RMSD change being related to the pressure and temperature (Table 1, Figure 1). As RMSD of the full structure is hardly to differentiate the local conformation, the residue root mean square fluctuations (RMSFs) were calculated to get the flexibility of the local domains. The structures, excluding the dangling $C$-terminal tail, presented similar structural features in all systems: the most mobile subdomain is the hairpin loop (L1) between $\beta 1$ and $\beta 2$ strands, whereas the residues in helices and $\beta$-sheets were less fluctuated (Figure S3). Such profile is generally consistent with the thermal motion quantified by the B-factor in crystal structure. The RMSF tendency with respect to pressure is promiscuous in L1, while in other subdomains the residue fluctuations in high pressure systems are usually not larger than in low pressure.

We rearranged the RMSF presentation into average values based on the protein secondary structure and residue topology (Figure 2). The trend of temperature effect is clear. Despite higher temperature generally caused higher fluctuation, the largest RMSF difference between 278 and $323 \mathrm{~K}$ is $0.3 \AA$, which reproduces the strong thermal stability of ubiquitin. On the other hand, the pressure influence is ambiguous. In different temperature groups, the trends of pressure influence were not consistent, for instance, the linearity of correlation between RMSF and pressure in 293 $\mathrm{K}$ and $308 \mathrm{~K}$ is better than that in $278 \mathrm{~K}$ and $323 \mathrm{~K}$. The average RMSF in $\alpha$-helices is mildly reduced with the pressure ranging from 1 to 2500 bar, whereas in $\beta$-sheets and loops such trend was only observed in $293 \mathrm{~K}$ (Table S1). For backbone atoms both helices and sheets have lower RMSF than loops, and for side chain atoms sheets and loops have higher RMSF than helices. In summary the local rigidity of ubiquitin structure is ranked as helix $>$ sheet $>$ loop, and the fluctuation reduction in response to pressure is also ranked as helix $>$ sheet $>$ loop.

\section{Backbone $\mathrm{H}$-bond dynamics reproduced using $\mathbf{~} \mathbf{3} \boldsymbol{J}_{\mathrm{NC}}$ couplings}

At least 53 backbone hydrogen bonds were observed in all systems and 37 of them were very stable in the simulations (Table S2). To validate the performance of $\mathrm{C} 36 \mathrm{~m}$ in different thermodynamic conditions, the $J$-coupling across atoms $\mathrm{N}-\mathrm{H} \cdots \mathrm{O}=\mathrm{C}\left({ }^{\mathrm{h} 3} J_{\mathrm{NC}}\right)$ was calculated from MD simulations and compared with the measurements of 33 backbone H-bonds in NMR study ${ }^{29}$. The discrepancy of calculation from experimental value, i.e. $\Delta^{\mathrm{h} 3} J_{\mathrm{NC} \prime}={ }^{\mathrm{h} 3} J_{\mathrm{NC} \text {, calc }}-{ }^{\mathrm{h} 3} J_{\mathrm{NC} \prime \text {, exp }}$, was adopted to evaluate the agreement between MD simulations and experimental data. For each 
system the average of absolute differences $\left|\Delta^{\mathrm{h} 3} J_{\mathrm{NC}}\right|$ for measured H-bonds is lower than $0.1 \mathrm{~Hz}$, which suggests the productions were consistent with experiment (Figure 3A). Taking account of the fact that many NMR detected $J$ couplings are smaller than $0.5 \mathrm{~Hz}$, we took the experimental value as denominator and thus the averages of relative discrepancy become 17\% 27\%. As a general view, no evidence shows the systematic deviation in any specific temperature group, nor is the discrepancy going higher as pressure increasing. This indicates the $\mathrm{C} 36 \mathrm{~m} \mathrm{FF}$, which is parametrized at room temperature and pressure, performs equally well for protein simulations under the varied thermodynamic conditions in range of 278 323 $\mathrm{K}$ and 1 2500 bar.

Most discrepancies are distributed equally close to zero, but significant deviations were observed for a number of $\mathrm{H}$-bonds which contributed significantly on the average results. Setting $\pm 0.1 \mathrm{~Hz}$ as the upper and lower bounders, the H-bond strengths of K6-L67, R42-V70, L67-F4, K33-K29 and I61-L56 were mostly overestimated, while T7-K11 and L56-D21 were underestimated (Figure 3B). The reproduction of these H-bonds has a systematic discrepancy to NMR measurement in all systems. On the other hand, compared to the crystal structure 1UQB all the backbone H-bonds were well reproduced and kept stable in each system, indicated by $\mathrm{H} \cdots \mathrm{O}$ distances shorter than $2.4 \AA$ with small fluctuations (Figure S4). The hydrogen bonds in L1 domain breathed more frequently than other domains, in accord with the high residue RMSFs. The weak H-bond S65-Q62 was also maintained.

Although these H-bonds were very stable in simulations and the distances at high pressures were generally shorter than at low pressures, the relationship between the interaction strength and pressure is not straightforward. The Pearson correlation coefficient $(\rho)$ is adopted to evaluate the linear correlation between $\mathrm{H}$-bond strength and pressure. It indicates the linearity between $\mathrm{H}$ bond strength and pressure, but it does not supply the slope of the linear correlation. As shown in Figure 4, the H-bonds with positive correlation $(\rho>0)$ in three or four temperature groups are more than those with negative correlation, which indicates the interactions of most H-bonds were likely enhanced from the elevated pressure.

The H-bonds that have positive pressure correlation in all temperatures include F4-S65, V17-M1, D21-E18 I23-R54, E34-I30, L56-D21, S57-P19, I61-L56, E64-Q2, S65-Q62 and H68-I44. Those correlations are consistent with the sign of derivative $\partial{ }^{\mathrm{h} 3} J_{\mathrm{NC} /} \mid / \partial p$ reported in the NMR study ${ }^{29}$, except for D21-E18 and S65-Q62 which were not detected experimentally probably due to the weak signal. The only two cases with all negative correlations are M1-V17 and R72-Q40. They are the first and last backbone H-bonds and acting as the gate hydrogen bonds of $\beta 1 / \beta 2$ sheet $(\mathrm{N}$ terminal) and $\beta 3 / \beta 5$ sheet (C-terminal) respectively. Note that the two backbone H-bonds between residue M1 and V17 have opposite responses to pressure. This observation resembles the case of R42-V70 reported in NMR study, where V70-R42 has positive correlation whereas R42V70 is the opposite (Figure S5). 
The H-bonds I3-L15, K6-L67, A28-E24, K48-F45, L50-L43 and N60-S57 have negative response with pressure in at least three temperatures. Their derivatives $\left.\partial\right|^{\mathrm{h} 3} J_{\mathrm{NC}^{\prime}} \mid / \partial p$ however are not significant which suggests the destabilization of pressure on those H-bonds is limited. Except for A28-E24, K48-F45 and N60-S57 which have not been reported experimentally, the rest correlations are consistent with the NMR study. Similar consistency was also kept for the Hbonds which have $\rho>0$ in three temperatures. In contrast, the positive linear correlation for L69K6 in experiment was not observed in the simulation. The remaining $\mathrm{H}$-bonds have either random signs of $\rho$ or low $|\rho|$, and displayed no linear correlation in this study. Those H-bonds may be considered to be generally stable and not influenced by current thermodynamic conditions.

\section{Methyl motions were partially restricted}

The internal motions of protein measured by NMR relaxation often respond differently to the system thermodynamic conditions. To evaluate the effect of pressure on the methyl-bearing motion of the ubiquitin, the order parameters $\left(S^{2}\right)$ of side chain methyl group were calculated. Two data sets of NMR ${ }^{2} \mathrm{H}$ relaxation in $303 \mathrm{~K}$ under $1 \mathrm{~atm}^{41}$ and various ambient pressures (1, $400,800,1200,1600,2500$ bar) ${ }^{26}$ were taken as the references. Both experiments show the similar profile of $S^{2}$ distribution while some measured values have difference. The methyl groups with low $S^{2}$ bear more motion in global conformational equilibration. The reproduced order parameters for all methyl axes of side chain were basically consistent with the profile of NMR data (Figure 5). Such distribution of internal motion for methyl groups also has some similarity with residue RMSF, where L1 and $\beta 3$ are more mobile than others. The underestimation in some sites, such as $\mathrm{T} 7 \gamma 2$, T $9 \gamma 2$ and $\mathrm{I} 30 \delta$, was also observed in previous simulations of C36 in room conditions ${ }^{20}$, but the error magnitude in this study using C $36 \mathrm{~m}$ is smaller.

The NMR measurements indicate many methyl groups have increased $S^{2}$ values in response to the ambient pressure. For residue of low order parameters, they are usually more sensitive to this effect. Those groups may be attached to both solvent exposed residues, for instance L8 $\delta 1 / 2$ and I44 $\delta$, and buried residues such as L67 $\delta 1 / 2$. The calculated order parameters also increased at elevated pressure for most residues especially in $\beta$-sheets and loops. Some residues even overresponded to the pressure such that their internal motions reduced more significantly than the experimental measurements. Such overestimation of pressure effect however brings the magnitude of calculated $S^{2}$ in high pressure closer to NMR data, for instance, the methyl groups of $\mathrm{I} 3 \gamma 2 / \delta, \mathrm{T} 7 \gamma 2$, I1 $3 \delta$ and $\mathrm{I} 30 \delta$. There are some sites that calculation did not reproduce the correct trend, for instance, I13 $\gamma 2$, L $67 \delta 1 / 2$ and $\mathrm{V} 70 \gamma 1 / 2$, where calculated correlations are opposite to NMR report. For the residues of intrinsically less mobility $\left(S^{2}>0.8\right)$, especially those located in the $\alpha$-helix, the calculated order parameters were not sensitive to the ambient pressure, in agreement with experiment.

\section{Ubiquitin transition states in response to pressure}

\section{Pocket was compressed but not penetrated by water}

From 1 to 2500 bar ubiquitin structure bears a compression, however the conformational change of most local domains is subtle in simulations. Ubiquitin is a typical globule protein in which most hydrophilic residues are solvent accessible and most hydrophobic residues are buried and 
thus solvent inaccessible. We evaluated the deformation of ubiquitin inner part to check how elevated pressure influences its structure.

Among 28 hydrophobic amino acids in the sequence of ubiquitin 14 residues whose side-chains aggregate inward from the solvent accessible surface were identified to be the hydrophobic core. We monitored the distances between the centers of mass of those 14 hydrophobic side chains, and calculated the Pearson correlation between each distance change and the ambient pressures. The map of distance-pressure correlation from simulations at $308 \mathrm{~K}$ has been shown in Figure 6, and the results in the other three temperatures were in Figure S6. Despite the overall changes are subtle, their correlation with pressure is clear, as most correlation coefficients are negative and close to -1 . This suggests the pair-wise distances between most side-chains in hydrophobic core were decreased in the compression process, and many of such distance decrement has linear correlation with pressure. A few cases that had weak positive correlations $(0<\rho<0.5)$ indicate these distances were not homogenously compressed. Our analysis suggests that the pressure effect on protein shape might not be isotropic and the dimension of the hydrophobic core was mildly adjusted in response to the compression.

Since those residues aggregate in a compact formation, they bore very low RMSF compared with more exposed residues (Figure S3). The space of compressible pocket volume therefore is very limited. The steady reduction in distance matrix also indicates that no water molecule penetrated into those hydrophobic groups, so the van der Waal radii of residues were forced to be reduced. This suggests that in the simulations using C36m FF, ubiquitin will not be destabilized by water penetration as pressure is up to 2500 bar.

\section{Global hydrogen bonds had different features}

Additional to H-bonds between backbones, the donors/acceptors of side-chain were also involved in hydrogen bonds and contribute to the structural stability of ubiquitin. Due to the bigger mobility, more than 100 side-chain involved H-bonds were monitored in simulations, but most of them were transient based on the average effective occupancies over all systems. While 37 of 53 backbone H-bonds had more than $70 \%$ occupancy, the side-chain H-bonds were much weaker as only 21 cases were lasting more than $30 \%$ simulation time (Table S2).

Ubiquitin is abundant of charged residues where 11 bases and 11 acids from 41 hydrophilic residues are distributed over whole domains. Their functional groups participate in all the side chain - side chain and half of the backbone - side chain H-bonds in MD simulations, thereby bringing additional electrostatic interaction to those H-bonds. There are 5 salt bridges (H-bond between a base and an acid residue) in the crystal structure. During simulations they were ordered by the average occupancies as: K27-D52 (0.95), K11-E34 (0.59), K63-E64 (0.36), R54-E51 (0.28) and R72-D39 (0.21). While lysines kept the H-bonds stable, the arginines were more flexible and their original H-bonds were rotated to R54-D58 (0.58) and R74-D39 (0.33) respectively. The salt bridges compose the most stable H-bonds between side chains, despite of the mobility of arginine. 
For the side chain H-bonds whose average occupancy is greater than $30 \%$, the Pearson correlation coefficients between occupancy and pressure were calculated and presented in Figure 7A. The most stable K27-D52 was less affected by pressure, while the other salt bridges had almost negative correlation to pressure. The exception R54-D58 was interconverted with R54-E51 where R54 is located between E51 and D58, the correlations of these H-bonds therefore are therefore opposite. At lower temperature, R54-E51 got enhancement from pressure whereas at high temperature R54-D58 was stabilized by pressure. Besides salt bridges, the remaining H-bonds between side chains with considerable occupancy include T55-D58 and K6-T12, and they did not have clear trend of occupancy in response for pressure. In brief, H-bonds between side chains were rarely stabilized by pressure.

The other category of H-bonds formed between backbone and side chain atoms were more stable and abundant than inter side-chain H-bonds. The H-bonds are formed either between $\mathrm{N}-\mathrm{H}$ and side-chain oxygen or $\mathrm{C}=\mathrm{O}$ and side-chain hydrogen. Among the $\mathrm{H}$-bonds with considerable average occupancy, E18-D21 (0.91), E51-Y59 (0.97) and D58-T55 (0.57) with amide HN as donor had positive linear correlation with pressure, whereas S65-Q62 (0.87) and Q62-N60 (0.44) with carbonyl $\mathrm{O}$ as acceptor had negative correlation. The remaining H-bonds Q41-I36 (0.70), Q41-K27 (0.59) and K27-Q41 (0.55) formed a triad where Q41 was in the middle, and did not show homogenous correlation with pressure in all temperatures.

Some residues that interacted through backbone $\mathrm{H}$-bond had also side chains that formed $\mathrm{H}$ bonds with each other, although only a few of them had considerable occupancies (Table S3). E18-D21 had the donor and acceptor different from the backbone H-bonds D21-E18, and both have positive correlation with pressure (Figure 4, Figure 7). This can be considered to be a synergy between backbone and side chain H-bonds. For more cases the H-bonds often share the same backbone acceptor or donor, so the correlation between strength and pressure were opposite. A typical case is S65-Q62 where amide HN and hydroxyl HG1 of S65 interacted with O of Q62 respectively. The backbone $\mathrm{H}$-bond was weak in the starting structure because $\mathrm{N}-\mathrm{H}$ was repulsed by its side chain $\mathrm{O}-\mathrm{H}$. However as the pressure increased, the distance $\mathrm{N}-\mathrm{H} \cdots \mathrm{O}=\mathrm{C}$ was compacted meanwhile pushing OG-HG1 away. Therefore the former H-bond is enhanced while the latter weakened. This is an example of competition between backbone and side chain H-bonds.

Compared to the H-bonds between backbones, the H-bonds consisting of side chain atoms had less stability and more negative correlations between strength and pressure. Although some sidechain involved H-bonds were constantly stable in all systems, in general circumstance the H-bond stability concerning with the composition in response to the pressure can be ordered as, backbones > backbone - side chain > side chains. In other words, the H-bonds composed of side chains would bear the pressure destabilization before the helix or sheet determined backbone $\mathrm{H}$ bonds. 
As a feature of aqueously soluble protein, the water bridged $\mathrm{H}$-bonds are very abundant in the simulations of ubiquitin, though most of them were insignificant for the low occupancies (Table S2). The water bridged H-bond rarely occurs in backbone, and only L43-L50 and A46-T66 were constantly monitored and the rest of all required side chain atoms involved. More frequently they formed between the side chains with low sequence separation, such as the turn of loop and intrahelix. The stability of water bridged H-bonds generally were lower than direct H-bonds because the long-range movement of side chain makes larger possibility of $\mathrm{H}$-bond rotations from one site to its neighbors. The fast water exchange rate reduces the lifetime of H-bond events, so their occupancies are further decreased. Meanwhile since the occupancy variation among different systems is large, the linear correlations related to the pressure are weaker than other H-bonds (Figure 7B). 


\section{Discussion}

Inspired by available high-pressure NMR data for ubiquitin, we validated the transferability of the $\mathrm{C} 36 \mathrm{~m}$ protein force field with respect to pressure using MD simulations with LJ-PME method implemented. The ${ }^{\mathrm{h} 3} J_{\mathrm{NC}}$, coupling is sensitive to identify the H-bond strength as its value is reverse to the exponential of $\mathrm{HN} \cdots \mathrm{O}$ distance (Eq. 1). MD simulations reproduced the $J$ couplings of backbone hydrogen bonds. To explore the relationship between pressure and protein structure from a range of discontinuous data, the regression is a useful way. According to the Pearson correlation coefficient, hydrogen bonds have three situations with respect to elevated pressure, enhanced $(\rho>0)$, destabilized $(\rho<0)$ and unclear $(\rho \approx 0)$. The first two are straightforward, while the last one is more complicated because it may represent case of either no correlation or non-linear correlation. Due to the limited data points of pressure (6 or 10 in each temperature) fitting of complicated functions is uncertain, so we only consider the general correlation in this study. For the strong H-bonds with high occupancy and small variance $(>0.7 \pm$ 0.1 ) through all systems, low $\rho \mid$ value means no correlation with pressure. On the other hand, for the H-bonds with large variance, the correlation of low $\rho \mid$ cannot be solved.

The pressure effect on backbone H-bonds was fully reproduced where the deviations of most reproduced ${ }^{\mathrm{h} 3} J_{\mathrm{NC}}$, from NMR data are lower than $0.1 \mathrm{~Hz}$. Large discrepancy systematically happened in seven sites, but five of them fit the $\mathrm{H} \cdots \mathrm{O}$ distance well, referring to the crystal conformation. Admittedly the crystal structure is only comparable to the NMR measurement at 1 bar, and the small difference does exist between two sets of experiments. ${ }^{42}$ Combining both experiments as the reference, two H-bonds with constant deviation are I61-L56 (overstated) and T7-K11 (underestimated). I61-L56 is a loop H-bond across $3_{10}$-helix and T7-K11 is the first Hbond of $\beta 1 / \beta 2$ hairpin, and both were influenced by the flexible neighbor residues in loops. Importantly such deviation is specific to certain H-bond pairs and is neither temperature nor pressure dependent. For this sake we may still conclude C36m FF is capable of the simulations in a large range of $p-T$ conditions.

Sensitivity in response to the ambient pressure is different for secondary structure elements. As the linear regression of average RMSF shows, the RMSFs in helices have negative coefficients under all temperatures other than sheets and loops (Table S1). The coefficients of determination $\left(R^{2}\right)$ varied largely among temperature groups, and only the systems in $293 \mathrm{~K}$ show relatively good linear correlation for all domains. This is probably due to the insufficient values of pressure considered. In $293 \mathrm{~K}$, the RMSFs of helices, sheets and loops are equivalently decreased with respect to pressure. Furthermore the Pearson correlation coefficients of H-bonds between helices and loops are mostly positive, where only A28-E24 and N60-S57 showed considerable negative correlations. This suggests the H-bonds not in sheets are more easily to be compacted. Those observations are similar with that in NMR study on pancreatic tyrosine inhibitor, where the helices and loops have more compressibility than sheets. ${ }^{43}$ 
The backbone H-bonds with negative linear correlation in all temperatures are M1-V17 $\left(\mathrm{N}^{+} \mathrm{H}_{3} \cdots \mathrm{O}\right)$ between $\beta 1 / \beta 2$ and R72-Q40 between $\beta 3 / \beta 5$. They were presented as the end $\mathrm{H}$-bonds from $\mathrm{N}$ - and $\mathrm{C}$-terminal respectively. In the case that ${ }^{\mathrm{h} 3} \mathrm{~J}_{\mathrm{NC}}$ of both M1-V17 and R72-Q40 were not presented experimentally, I3-L15 and R42-V70 were the first and last H-bonds respectively in NMR structures and the strength of R42-V70 is negatively correlated with pressure. ${ }^{29,42}$ According to the hypothesis from these NMR observations, the pressure denaturation of ubiquitin begins at position of R42-V70, the gate of $\beta 3 / \beta 5$ strand. The phenomenon reproduced on R72Q40 in simulations is largely consistent with R42-V70 in experiments, where the gate H-bond bears the destabilization but its neighbor is not influenced. In addition, the simulation results indicate the pressure destabilization could also initiate on the gate $\mathrm{H}$-bond of ionized N-terminal.

We also studied the pressure effects on side-chain involved H-bonds, which have not been systematically reported in experiments. Effective occupancy was computed for those H-bonds instead of the through-space $J$ couplings. Charged residues are the major contributors to the sidechain involved H-bonds, so the extra electrostatic attraction might enhance interaction energy between donor and acceptor. However while most H-bonds between backbones were stabilized by elevated pressure, the H-bonds between side chains were mostly destabilized except for a few salt-bridges which were not influenced. The results that backbones involved H-bonds have better stabilization in response to pressure than side chains suggest the dynamic of side chain should be a more dominant factor than charge attraction to the maintenance of H-bonds in simulations.

The flexibilities of direct and water bridged H-bonds are also different in response to pressure. The lifetime of H-bond events varied from several picoseconds to hundreds of nanoseconds in long MD simulation. For direct H-bonds, the shorter events (lifetime $\leq 6 \mathrm{~ns}$ ) are decreased along with the elevation of pressure, while the longer events ( $6 \mathrm{~ns}<$ lifetime $\leq 100 \mathrm{~ns}$ ) are increased (data not shown). This is reasonable that the breath frequency of some H-bonds is reduced as the distances between donor and acceptor are compressed. The exception is for a few constantly long events (lifetime > $100 \mathrm{~ns}$ ), which are originally rigid and not influenced by the pressure. In general pressure reduces the dynamics of global inter-residue H-bonds and elongates the average lifetimes of the interaction.

The number of water-bridged H-bonds is significantly larger, but most of these H-bonds are transient as lifetimes being shorter than $4 \mathrm{~ns}$. Those short events are increased with respect to the hydrostatic pressure rising up. Compared to direct H-bonds, more H-bonds have been formed between residues and water molecules but fewer H-bonds get reduced breath frequency to be more stable. This consistent with the observation of another computational work for short peptide and TIP4P water model, where the H-bonds between backbone and solvent are more dynamic in high pressure at $300 \mathrm{~K}$ thereby producing more short events. ${ }^{44}$ Elevated pressures increase the probability of $\mathrm{H}$-bonds between water and protein surface residues by compressing their atomic radii. Yet at 2500 bar, there is still no extra cavity caused by water penetration according to the hypothesis of pressure denaturation proposed in another MD simulation. ${ }^{45}$ 
Water bridged contact between hydrophobic side-chain depicts the non-polar interaction between solvent and protein. Totally 24 water bridged contacts were observed and they are only located on solvent exposed residues, i.e., no water was observed between the side chains of the 14 residues that is defined as hydrophobic core. This indicates that there is no water penetration as the volume hydrophobic core compresses under pressure, in agreement with the NMR conclusion that up to 2500 bar ubiquitin is stable. ${ }^{26,29}$ Similar to H-bonds, the events of water bridged contact are tremendously increased with pressure as the distance between water and hydrophobic side chains are also compressed. However since no requirement of interactive direction when water is approaching to the hydrophobic groups in the compression, such non-polar contact has stronger linear correlation with pressure than $\mathrm{H}$-bonds (Figure 7C). In summary both water bridged $\mathrm{H}$ bonds and contacts confirm the access radius of surface atoms is reduced in response to hydrostatic pressure while maintaining the conformation.

Several computational studies have studied the water penetration into the ubiquitin hydrophobic core as the ambient pressure increased up to 3000 bar using Amber $94^{45}$ based on the ensemble in NMR study, ${ }^{46}$ and up to 10000 bar using CHARMM22 with a random walk sampling in pressure space. ${ }^{47}$ Their simulations suppose the mechanism of pressure denaturation through the energetic profile of solvation shell, in which the water entering hydrophobic core is the most important transition state for structural deformation. Indeed the infrared spectroscopy shows that the ubiquitin unfolding occurs at 5400 bar, where the secondary structures were rearranged rather than disordered. ${ }^{48}$ The prediction of pressure destabilization that extends the phase diagram of pressure from native to denatured state is out of the scope in this study. Instead, by reproducing the experimental $\mathrm{H}$-bonds in response to hydrostatic pressure, we identified the ubiquitin transition states in which the gate $\mathrm{H}$-bonds in sheets and the H-bonds between side chains firstly bear the pressure destabilization more than others. The structure is stable up to $2500 \mathrm{bar}$, but the polar and non-polar contacts between residue and water molecule are further increased because of the compression. 


\section{Conclusion}

The major purpose of this study is to evaluate the capability of CHARMM36m additive force field combined with the LJ-PME nonbonded method in a wide range of temperature and pressure. By reproducing the backbone hydrogen bonds and the internal motion of methyl groups from 1 to 2500 bar based on long MD simulations, the calculated properties are in good agreement with NMR observations. The deviations are independent of the temperature and pressure of the simulation systems. These results verified that protein force field CHARMM36m has good transferability in MD simulations with different conditions of pressure.

Following the experimental data, the study also explored the transition states of ubiquitin under the elevated pressure. The pressure generally reduced the residue flexibility and helices are more sensitive than sheets and loops. Except for gate H-bonds between sheets whose strength is weakened, most $\mathrm{H}$-bonds between backbones are either enhanced or not influenced in response to pressure. In contrast, the side chain involved H-bonds are more fragile to bear the pressure destabilization, which indicates that the pressure denaturation starts from the side-chain H-bonds and then the backbone between sheets. Pressure reduces fluctuation of inter-residue H-bonds meanwhile increases both the hydrophilic and hydrophobic contacts by compressing the atomic radii. However up to 2500 bar, no cavity is formed to enable water penetration in ubiquitin. The feasibility of modern force fields combined with LJ-PME would encourage more focus on the computational study on biomolecules under elevated pressure.

\section{Acknowledgement}

The work is supported by Zhejiang Provincial Natural Science Foundation of China (Grant No. LR19B030001 and LQ20C050001), National Natural Science Foundation of China (Grant No. 21803057), Westlake Education Foundation and Tencent Foundation. We thank Westlake University Supercomputer Center for computational resource and related assistance. 


\section{Tables}

Table 1. The $p$ - $T$ conditions that were adopted in $1 \mathrm{UQB}$ simulations. The values show the average RMSD $(\AA)$ of heavy atom coordinates calculated over $1.2 \mu$ s for each system, and the systems involved in the NMR experiment ${ }^{29}$ are shaded.

\begin{tabular}{|l|c|c|c|c|c|c|c|c|c|c|}
\hline $\begin{array}{c}R \text { (bar) } \\
T(\mathrm{~K})\end{array}$ & 1 & 300 & 500 & 600 & 900 & 1000 & 1200 & 1500 & 2000 & 2500 \\
\hline 278 & 1.76 & & 1.76 & & & 1.94 & & 1.78 & 1.79 & 1.74 \\
\hline 293 & 1.83 & & 1.81 & & & 1.81 & & 1.87 & 1.77 & 1.79 \\
\hline 308 & 1.75 & 1.71 & 1.79 & 1.80 & 1.79 & 1.76 & 1.79 & 1.76 & 1.80 & 1.81 \\
\hline 323 & 1.80 & & 1.74 & & & 1.81 & & 1.80 & 1.97 & 1.82 \\
\hline
\end{tabular}


Table 2. Correlation between experimental and calculated ${ }^{\mathbf{h} 3} \boldsymbol{J}_{\mathrm{NC}}$ couplings in five proteins. The 1000 ns MD trajectories were partitioned into ten $100 \mathrm{~ns}$ blocks, and the correlation coefficient, RMSD and Q factors for each block were computed. Average correlation coefficients, RMSD and Q factors and their standard errors are obtained from simulations using the $\mathrm{C} 36 \mathrm{~m}$ protein force field with and without LJ-PME, respectively.

\begin{tabular}{lcccccc}
\multirow{2}{*}{ proteins } & \multicolumn{2}{c}{ correlation coefficient } & \multicolumn{2}{c}{ RMSD $(\mathrm{Hz})$} & \multicolumn{2}{c}{ Q factor } \\
\cline { 2 - 7 } & cutoff & LJ-PME & cutoff & LJ-PME & cutoff & LJ-PME \\
\hline 1UBQ & $0.81 \pm 0.01$ & $0.78 \pm 0.01$ & $0.109 \pm 0.002$ & $0.109 \pm 0.003$ & $0.20 \pm 0.00$ & $0.21 \pm 0.01$ \\
2QMT & $0.78 \pm 0.01$ & $0.75 \pm 0.02$ & $0.119 \pm 0.001$ & $0.128 \pm 0.007$ & $0.24 \pm 0.00$ & $0.27 \pm 0.02$ \\
1MJC & $0.74 \pm 0.01$ & $0.76 \pm 0.01$ & $0.141 \pm 0.002$ & $0.142 \pm 0.003$ & $0.27 \pm 0.01$ & $0.27 \pm 0.01$ \\
1QX5 & $0.23 \pm 0.02$ & $0.33 \pm 0.02$ & $0.189 \pm 0.005$ & $0.169 \pm 0.004$ & $0.43 \pm 0.01$ & $0.38 \pm 0.01$ \\
1IFC & $0.69 \pm 0.01$ & $0.68 \pm 0.01$ & $0.163 \pm 0.002$ & $0.167 \pm 0.002$ & $0.29 \pm 0.00$ & $0.29 \pm 0.00$
\end{tabular}




\section{Figures}

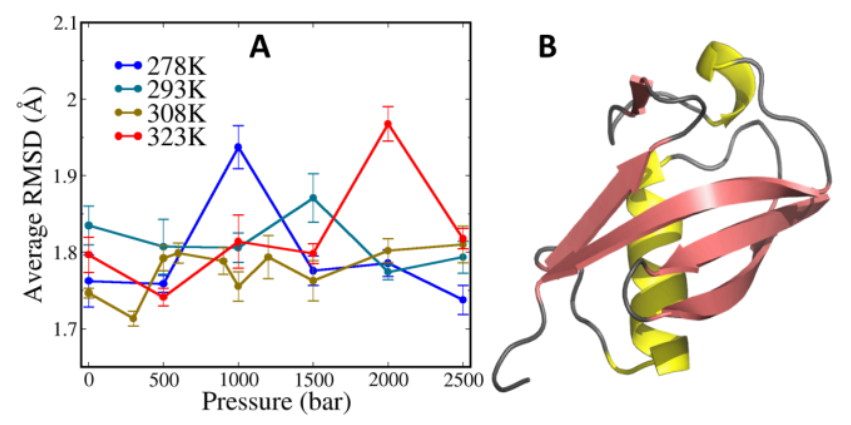

Figure 1. The overview of the simulation. A. RMSD from crystal structure of heavy-atom coordinates calculated in each system. Each value was averaged from the last $1 \mu$ s simulation with the block standard deviation shown as the error bar. B, The 3-D structure of 1UBQ with yellow for helices, pink for sheets and gray for loops. 


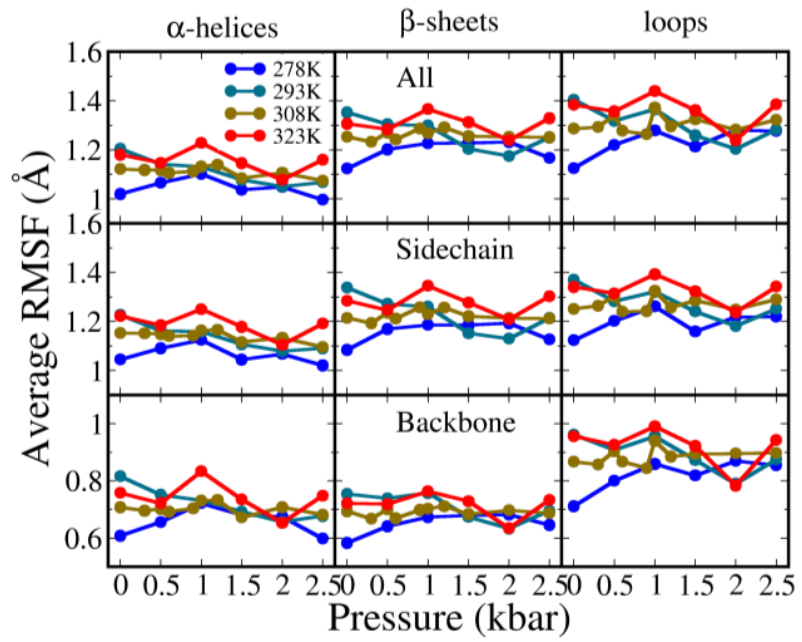

Figure 2. The average RMSF of heavy atoms in all systems. Three rows show the data of all atoms, sidechain atoms and backbone atoms, respectively, and three columns for helices, sheets and loops, respectively. 

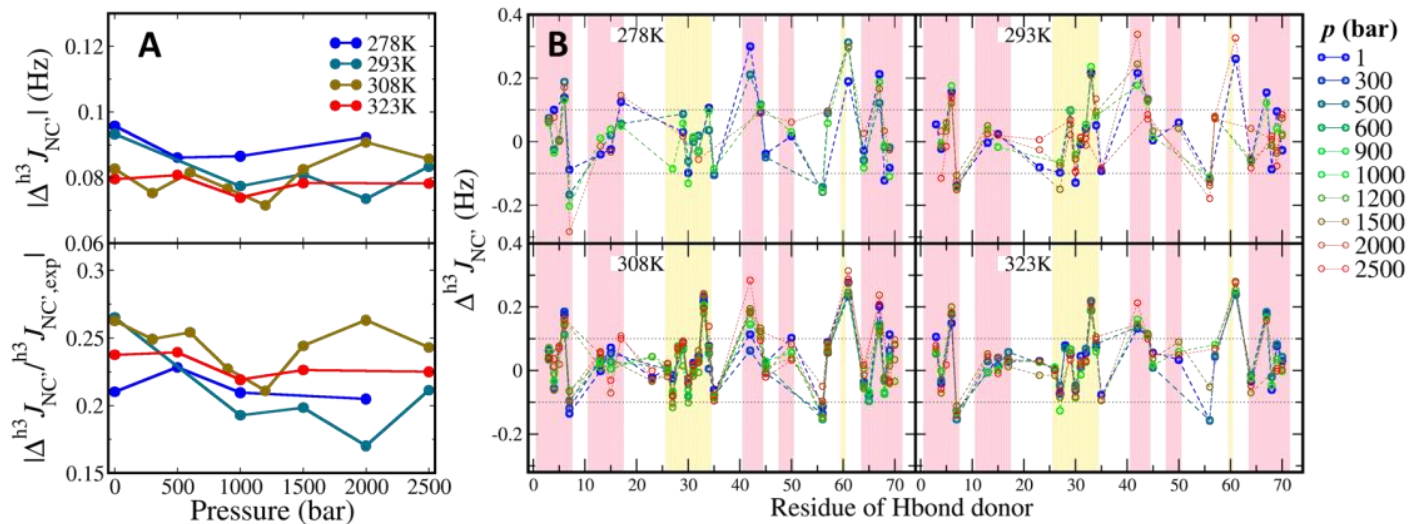

Figure 3. The discrepancy of calculated ${ }^{\mathrm{h} 3} \boldsymbol{J}_{\mathrm{NC}}$, coupling from the experiments. A. The absolute average ${ }^{\mathrm{h} 3} J_{\mathrm{NC}}$, difference of each system. The upper panel shows the average of absolute difference and the lower panel shows the ratio of absolute difference over experimental value. B. The residue-wise $\Delta^{\mathrm{h} 3} J_{\mathrm{NC}}$, of all systems. Each panel organizes the $\Delta^{\mathrm{h} 3} J_{\mathrm{NC}}$ of different pressures in a specific temperature. The hydrogen bonds are indexed by the donor residue. The panel background is colored by the secondary structure domains, i.e. yellow for helices and pink for sheets. The dotted baselines indicate the thresholds $\pm 0.1 \mathrm{~Hz}$. 


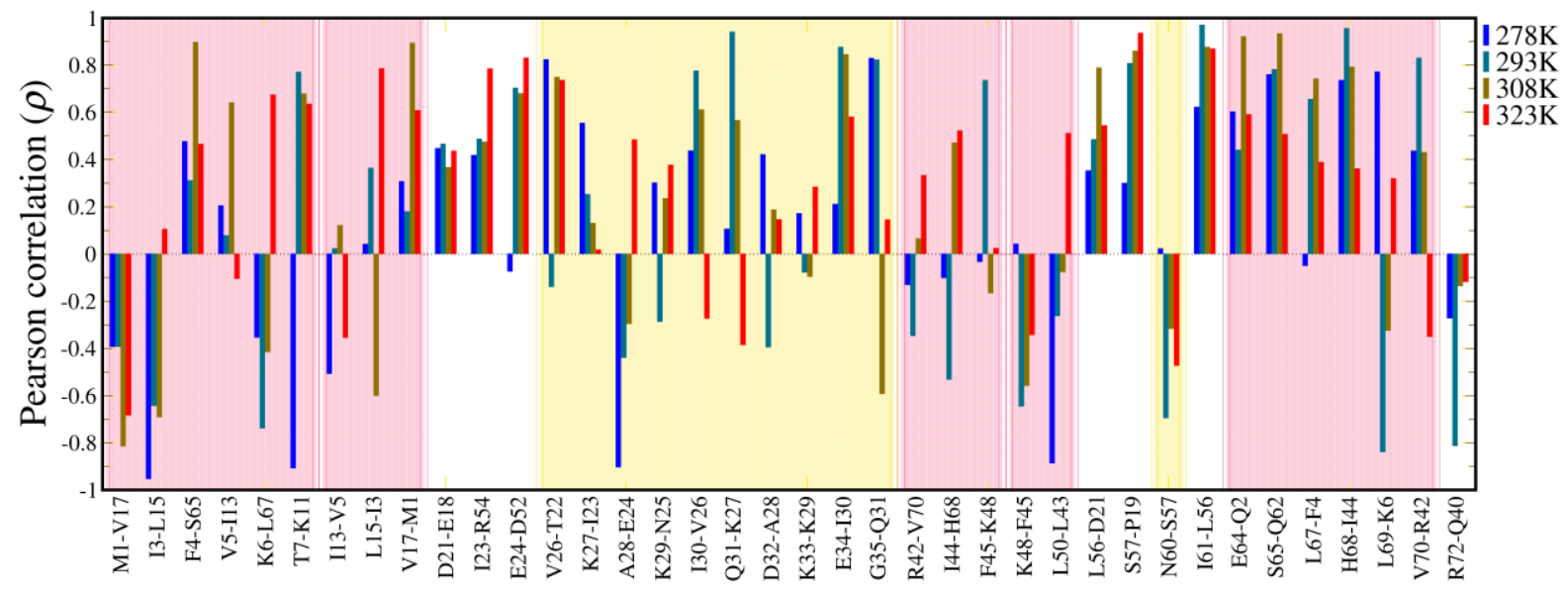

Figure 4. Pearson correlation between ${ }^{\mathrm{h} 3} \mathrm{~J}_{\mathrm{NC}}$ of backbone $\mathrm{H}$-bond and hydrostatic pressure. The dashed line at $\rho=0$ indicates no linear correlation at all, while $\rho=1$ and $\rho=-1$ indicate totally positive and negative linear correlation respectively. 


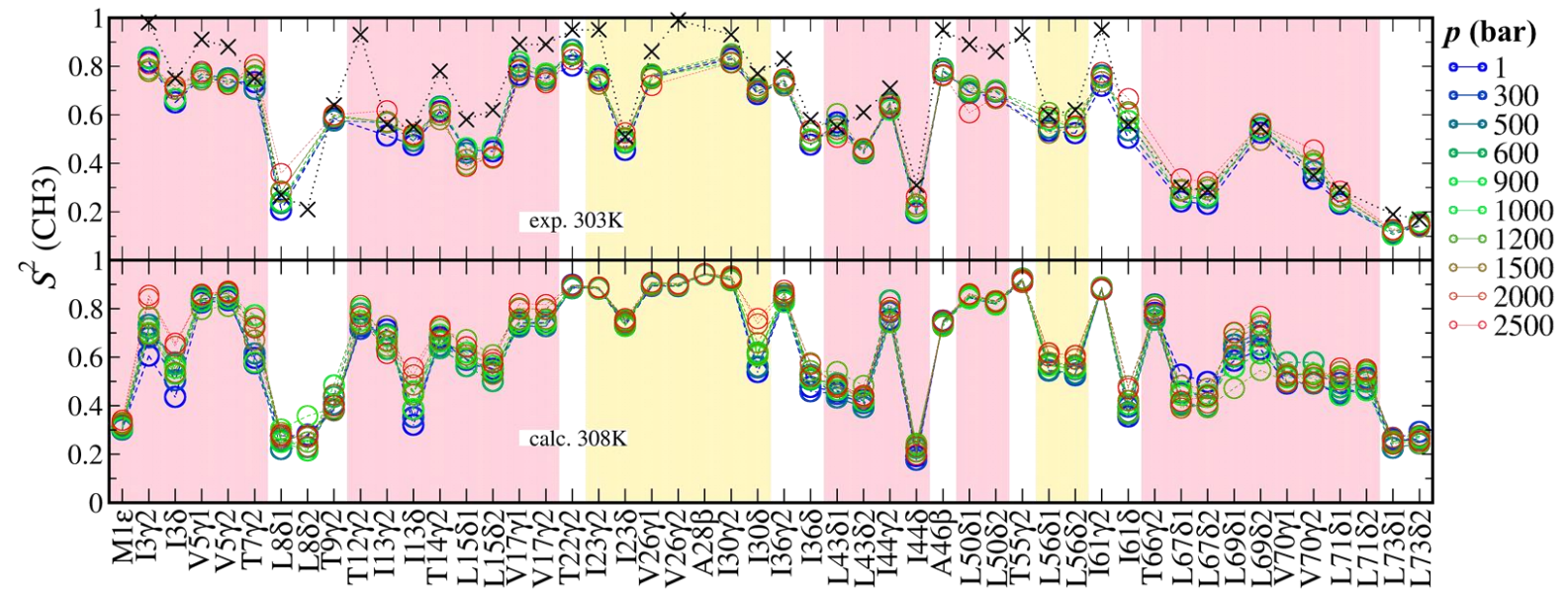

Figure 5. Order parameter $\left(S^{2}\right)$ of side chain methyl group in ubiquitin. The $x$-axis is indexed by the methyl carbon name of the residues. The upper panel shows the data from two experiments (circles with ambient pressures ${ }^{26}$ and crosses at $1 \mathrm{~atm}^{41}$ ) at $303 \mathrm{~K}$ and the lower shows calculated data at $308 \mathrm{~K}$. 


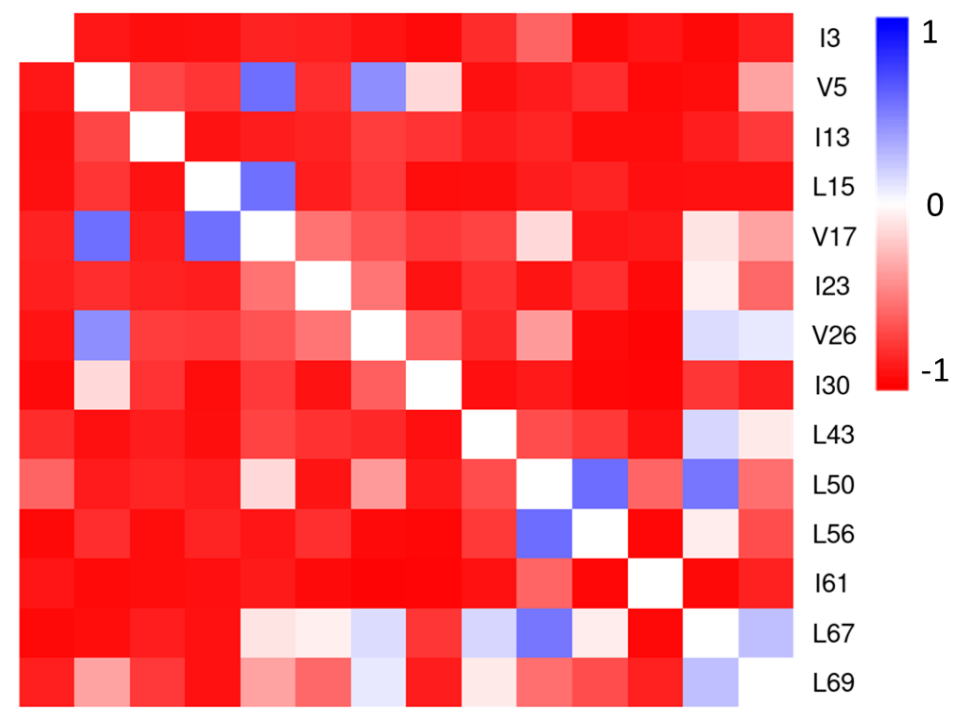

$\begin{array}{lllllllllllllll}\text { I3 } & \text { V5 } & \text { I13 L15 V17 } & \text { I23 V26 } & \text { I30 } & \text { L43 } & \text { L50 } & \text { L56 } & \text { I61 } & \text { L67 } & \text { L69 }\end{array}$

Figure 6. Pearson correlation coefficients between hydrophobic distances and pressure at $308 \mathrm{~K}$. The pairwise distances were measured between the mass centers of 14 hydrophobic side-chains in the pocket. From $\rho=1$ (blue) to $\rho=-1$ (red), the distances correlate with pressure from positively to negatively. 


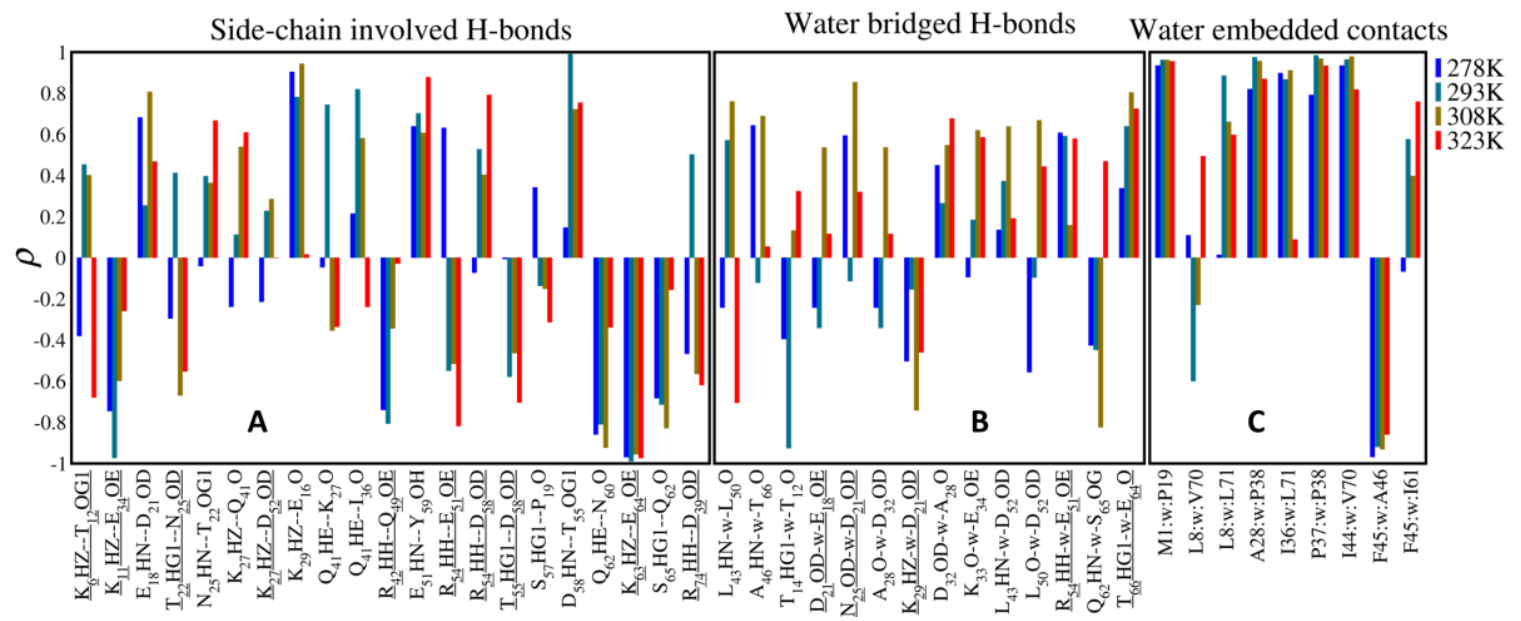

Figure 7. Pearson correlation between the effective occupancy and pressure for hydrogen bonds or hydrophobic contacts. A) The donors/acceptors of side-chain involved direct $\mathrm{H}$ - bonds. B) The water bridged H-bonds. The $x$-axis labels both specific residue and atom involved in the $\mathrm{H}$-bonds, and the ones with underline indicate the $\mathrm{H}$-bonds between side chains and the others are between backbone and side chain. C) The water embedded hydrophobic contacts. 


\section{References}

1. Luong, T. Q.; Erwin, N.; Neumann, M.; Schmidt, A.; Loos, C.; Schmidt, V.; Fandrich, M.; Winter, R., Hydrostatic Pressure Increases the Catalytic Activity of Amyloid Fibril Enzymes.

Angew. Chem. Int. Edit. 2016, 55, 12412-12416.

2. Stiller, J. B.; Kerns, S. J.; Hoemberger, M.; Cho, Y. J.; Otten, R.; Hagan, M. F.; Kern, D., Probing the transition state in enzyme catalysis by high-pressure NMR dynamics. Nature Catalysis 2019, 2, 726-734.

3. Sun, X. D.; Holley, R. A., High Hydrostuatic Pressure Effects on the Texture of Meat and Meat Products. J. Food Sci. 2010, 75, R17-R23.

4. Laroussi, M., Low temperature plasma-based sterilization: Overview and state-of-the-art. Plasma Processes and Polymers 2005, 2, 391-400.

5. Considine, K. M.; Kelly, A. L.; Fitzgerald, G. F.; Hill, C.; Sleator, R. D., High-pressure processing - effects on microbial food safety and food quality. FEMS Microbiol. Lett. 2008, 281, $1-9$.

6. Wu, E. L.; Cheng, X.; Jo, S.; Rui, H.; Song, K. C.; Davila-Contreras, E. M.; Qi, Y. F.; Lee, J. M.; Monje-Galvan, V.; Venable, R. M.; Klauda, J. B.; Im, W., CHARMM-GUI Membrane Builder Toward Realistic Biological Membrane Simulations. J. Comput. Chem. 2014, 35, 1997-2004.

7. Qi, Y. F.; Ingolfsson, H. I.; Cheng, X.; Lee, J.; Marrink, S. J.; Im, W., CHARMM-GUI Martini Maker for Coarse-Grained Simulations with the Martini Force Field. J. Chem. Theory Comput. 2015, 11, 4486-4494.

8. Dignon, G. L.; Zheng, W. W.; Best, R. B.; Kim, Y. C.; Mittal, J., Relation between singlemolecule properties and phase behavior of intrinsically disordered proteins. Proc. Natl. Acad. Sci. U.S.A. 2018, 115, 9929-9934.

9. Cinar, H.; Fetahaj, Z.; Cinar, S.; Vernon, R. M.; Chan, H. S.; Winter, R. H. A., Temperature, Hydrostatic Pressure, and Osmolyte Effects on Liquid-Liquid Phase Separation in Protein Condensates: Physical Chemistry and Biological Implications. Chemistry-a European Journal 2019, 25, 13049-13069.

10. Feig, M.; Sugita, Y., Variable Interactions between Protein Crowders and Biomolecular Solutes Are Important in Understanding Cellular Crowding. J. Phys. Chem. B 2012, 116, 599-605. 11. Harada, R.; Sugita, Y.; Feig, M., Protein Crowding Affects Hydration Structure and Dynamics. Journal of the American Chemical Society 2012, 134, 4842-4849.

12. Jones, H. B. L.; Wells, S. A.; Prentice, E. J.; Kwok, A.; Liang, L. L.; Arcus, V. L.; Pudney, C. R., A complete thermodynamic analysis of enzyme turnover links the free energy landscape to enzyme catalysis. FEBS J. 2017, 284, 2829-2842.

13. Cinar, S.; Al-Ayoubi, S.; Sternemann, C.; Peters, J.; Winter, R.; Czeslik, C., A high pressure study of calmodulin-ligand interactions using small-angle $\mathrm{X}$-ray and elastic incoherent neutron scattering. Physical Chemistry Chemical Physics 2018, 20, 3514-3522.

14. Charlier, C.; Alderson, T. R.; Courtney, J. M.; Ying, J. F.; Anfinrud, P.; Bax, A., Study of protein folding under native conditions by rapidly switching the hydrostatic pressure inside an NMR sample cell. Proc. Natl. Acad. Sci. U.S.A. 2018, 115, E4169-E4178.

15. Roche, J.; Royer, C. A., Lessons from pressure denaturation of proteins. Journal of the Royal Society Interface 2018, 15.

16. Ichiye, T., What makes proteins work: exploring life in P-T-X. Phys Biol 2016, 13.

17. Huang, Q.; Tran, K. N.; Rodgers, J. M.; Bartlett, D. H.; Hemley, R. J.; Ichiye, T., A molecular perspective on the limits of life: Enzymes under pressure. Condens. Matter Phys. 2016, 19. 
18. Beauchamp, K. A.; Lin, Y. S.; Das, R.; Pande, V. S., Are Protein Force Fields Getting Better? A Systematic Benchmark on 524 Diverse NMR Measurements. J. Chem. Theory Comput. 2012, 8, $1409-1414$.

19. Huang, J.; Meuwly, M., Explicit Hydrogen-Bond Potentials and Their Application to NMR Scalar Couplings in Proteins. J. Chem. Theory Comput. 2010, 6, 467-476.

20. Huang, J.; MacKerell, A. D., Jr., CHARMM36 all-atom additive protein force field: validation based on comparison to NMR data. J. Comput. Chem. 2013, 34, 2135-45.

21. Lange, O. F.; van der Spoel, D.; de Groot, B. L., Scrutinizing Molecular Mechanics Force Fields on the Submicrosecond Timescale with NMR Data. Biophys. J. 2010, 99, 647-655.

22. Cordier, F.; Grzesiek, S., Direct observation of hydrogen bonds in proteins by interresidue (3h)J(NC') scalar couplings. Journal of the American Chemical Society 1999, 121, 1601-1602.

23. Cornilescu, G.; Hu, J. S.; Bax, A., Identification of the hydrogen bonding network in a protein by scalar couplings. Journal of the American Chemical Society 1999, 121, 2949-2950.

24. Huang, J.; Rauscher, S.; Nawrocki, G.; Ran, T.; Feig, M.; de Groot, B. L.; Grubmuller, H.; Mackerell, A. D., Jr., CHARMM36m: an improved force field for folded and intrinsically disordered proteins. Nat. Methods 2017, 14, 71-73.

25. Kuzmanic, A.; Pritchard, R. B.; Hansen, D. F.; Gervasio, F. L., Importance of the Force Field Choice in Capturing Functionally Relevant Dynamics in the von Willebrand Factor. J. Phys. Chem. Lett. 2019, 10, 1928-1934.

26. Fu, Y. N.; Kasinath, V.; Moorman, V. R.; Nucci, N. V.; Hilser, V. J.; Wand, A. J., Coupled Motion in Proteins Revealed by Pressure Perturbation. Journal of the American Chemical Society 2012, 134, 8543-8550.

27. Krishnan, M.; Smith, J. C., Reconstruction of Protein Side-Chain Conformational Free Energy Surfaces From NMR-Derived Methyl Axis Order Parameters. J. Phys. Chem. B 2012, 116, 4124-4133.

28. Best, R. B.; Clarke, J.; Karplus, M., What contributions to protein side-chain dynamics are probed by NMR experiments? A molecular dynamics simulation analysis. J. Mol. Biol. 2005, 349, 185-203.

29. Nisius, L.; Grzesiek, S., Key stabilizing elements of protein structure identified through pressure and temperature perturbation of its hydrogen bond network. Nature chemistry 2012, 4, 711-7.

30. Shirts, M. R.; Mobley, D. L.; Chodera, J. D.; Pande, V. S., Accurate and efficient corrections for missing dispersion interactions in molecular Simulations. J. Phys. Chem. B 2007, 111, 13052-13063.

31. Wennberg, C. L.; Murtola, T.; Hess, B.; Lindahl, E., Lennard-Jones Lattice Summation in Bilayer Simulations Has Critical Effects on Surface Tension and Lipid Properties. J. Chem. Theory Comput. 2013, 9, 3527-3537.

32. Wennberg, C. L.; Murtola, T.; Pall, S.; Abraham, M. J.; Hess, B.; Lindahl, E., Direct-Space Corrections Enable Fast and Accurate Lorentz-Berthelot Combination Rule Lennard-Jones Lattice Summation. J. Chem. Theory Comput. 2015, 11, 5737-5746.

33. Leonard, A. N.; Simmonett, A. C.; Pickard, F. C.; Huang, J.; Venable, R. M.; Klauda, J. B.; Brooks, B. R.; Pastor, R. W., Comparison of Additive and Polarizable Models with Explicit Treatment of Long-Range Lennard-Jones Interactions Using Alkane Simulations. J. Chem. Theory Comput. 2018, 14, 948-958.

34. Vijay-Kumar, S.; Bugg, C. E.; Cook, W. J., Structure of ubiquitin refined at 1.8 A resolution. J. Mol. Biol. 1987, 194, 531-44. 
35. Jorgensen, W. L.; Chandrasekhar, J.; Madura, J. D.; Impey, R. W.; Klein, M. L., Comparison of Simple Potential Functions for Simulating Liquid Water. J. Chem. Phys. 1983, 79, 926-935.

36. Darden, T.; York, D.; Pedersen, L., Particle Mesh Ewald - an N.Log(N) Method for Ewald Sums in Large Systems. J. Chem. Phys. 1993, 98, 10089-10092.

37. Best, R. B.; Zhu, X.; Shim, J.; Lopes, P. E. M.; Mittal, J.; Feig, M.; MacKerell, A. D., Optimization of the Additive CHARMM All-Atom Protein Force Field Targeting Improved Sampling of the Backbone phi, psi and Side-Chain chi(1) and chi(2) Dihedral Angles. J. Chem. Theory Comput. 2012, 8, 3257-3273.

38. Eastman, P.; Swails, J.; Chodera, J. D.; McGibbon, R. T.; Zhao, Y. T.; Beauchamp, K. A.; Wang, L. P.; Simmonett, A. C.; Harrigan, M. P.; Stern, C. D.; Wiewiora, R. P.; Brooks, B. R.; Pande, V. S., OpenMM 7: Rapid development of high performance algorithms for molecular dynamics. PLoS Comp. Biol. 2017, 13, e1005659.

39. Brooks, B. R.; Brooks, C. L., 3rd; Mackerell, A. D., Jr.; Nilsson, L.; Petrella, R. J.; Roux, B.; Won, Y.; Archontis, G.; Bartels, C.; Boresch, S.; Caflisch, A.; Caves, L.; Cui, Q.; Dinner, A. R.; Feig, M.; Fischer, S.; Gao, J.; Hodoscek, M.; Im, W.; Kuczera, K.; Lazaridis, T.; Ma, J.; Ovchinnikov, V.; Paci, E.; Pastor, R. W.; Post, C. B.; Pu, J. Z.; Schaefer, M.; Tidor, B.; Venable, R. M.; Woodcock, H. L.; Wu, X.; Yang, W.; York, D. M.; Karplus, M., CHARMM: the biomolecular simulation program. J. Comput. Chem. 2009, 30, 1545-614.

40. Barfield, M., Structural dependencies of interresidue scalar coupling (h3)J(NC), and donor $\mathrm{H}-1$ chemical shifts in the hydrogen bonding regions of proteins. Journal of the American Chemical Society 2002, 124, 4158-4168.

41. Lee, A. L.; Flynn, P. F.; Wand, A. J., Comparison of H-2 and C-13 NMR relaxation techniques for the study of protein methyl group dynamics in solution. Journal of the American Chemical Society 1999, 121, 2891-2902.

42. Cordier, F.; Grzesiek, S., Temperature-dependence of protein hydrogen bond properties as studied by high-resolution NMR. J. Mol. Biol. 2002, 317, 739-52.

43. Akasaka, K.; Li, H.; Yamada, H.; Li, R. H.; Thoresen, T.; Woodward, C. K., Pressure response of protein backbone structure. Pressure-induced amide N-15 chemical shifts in BPTI. Protein Sci. 1999, 8, 1946-1953.

44. Nellas, R. B.; Glover, M. M.; Hamelberg, D.; Shen, T. Y., High-pressure effect on the dynamics of solvated peptides. J. Chem. Phys. 2012, 136.

45. Day, R.; Garcia, A. E., Water penetration in the low and high pressure native states of ubiquitin. Proteins 2008, 70, 1175-1184.

46. Kitahara, R.; Yokoyama, S.; Akasaka, K., NMR snapshots of a fluctuating protein structure: Ubiquitin at 30 bar-3 kbar. J. Mol. Biol. 2005, 347, 277-285.

47. Mori, Y.; Okamoto, Y., Conformational changes of ubiquitin under high pressure conditions: A pressure simulated tempering molecular dynamics study. J. Comput. Chem. 2017, 38, 1167-1173.

48. Herberhold, H.; Winter, R., Temperature- and pressure-induced unfolding and refolding of ubiquitin: A static and kinetic Fourier transform infrared spectroscopy study. Biochemistry 2002, 41, 2396-2401. 


\section{Supporting information}

Protein Stabilization Using Molecular Simulation in Pressure and Temperature Conditions

$$
\text { You } \mathrm{Xu}^{1,2,3} \text {, Jing Huang }{ }^{1,2,3 *}
$$

1. Key Laboratory of Structural Biology of Zhejiang Province, School of Life Sciences, Westlake University, 18 Shilongshan Road, Hangzhou, Zhejiang 310024, China.

2. Westlake Laboratory of Life Sciences and Biomedicine, 18 Shilongshan Road, Hangzhou, Zhejiang 310024, China.

3. Institute of Biology, Westlake Institute for Advanced Study, 18 Shilongshan Road, Hangzhou, Zhejiang 310024, China.

*Corresponding author: Jing Huang, huangjing@ westlake.edu.cn 


\section{Tables}

Table S1. Linear regression of average atomic RMSF with respect to the pressure. The slopes presented have been multiplied by 10000 .

\begin{tabular}{|l|l|l|l|l|l|l|}
\hline \multirow{2}{*}{$T(\mathrm{~K})$} & \multicolumn{2}{|c|}{$\alpha$-helices } & \multicolumn{2}{c|}{$\beta$-sheets } & \multicolumn{2}{c|}{ loops } \\
\cline { 2 - 7 } & $10^{4} a$ & $R^{2}$ & $10^{4} a$ & $R^{2}$ & $10^{4} a$ & $R^{2}$ \\
\hline 278 & -0.12 & 0.14 & 0.16 & 0.14 & 0.48 & 0.63 \\
\hline 293 & -0.46 & 0.75 & -0.58 & 0.63 & -0.56 & 0.48 \\
\hline 308 & -0.15 & 0.33 & 0.01 & 0 & 0.08 & 0.03 \\
\hline 323 & -0.21 & 0.17 & -0.04 & 0.01 & -0.19 & 0.09 \\
\hline
\end{tabular}

Table S2. Statistics of detected H-bonds in simulations with the average effective occupancy greater than $\mathbf{5 \%}$ over all temperatures and pressures.

\begin{tabular}{|l|l|l|l|l|l|l|l|l|l|l|l|l|}
\hline & \multicolumn{10}{|l|}{ Direct } \\
\hline Occupancy (\%) & Tot. & $>70$ & $>50$ & $>30$ & $>10$ & $>5$ & Tot. & $>70$ & $>50$ & $>30$ & $>10$ & $>5$ \\
\hline Backbones & 53 & 37 & 1 & 5 & 6 & 4 & 3 & 1 & 0 & 1 & 1 & 0 \\
\hline $\begin{array}{l}\text { Backbone - side } \\
\text { chain }\end{array}$ & 30 & 4 & 3 & 7 & 16 & 0 & 58 & 1 & 2 & 6 & 25 & 24 \\
\hline Side chains & 17 & 1 & 2 & 4 & 6 & 4 & 44 & 0 & 4 & 3 & 28 & 9 \\
\hline
\end{tabular}

Table S3. The number of residue duet that has side chain involved H-bonds additional to backbone H-bonds.

\begin{tabular}{|l|l|l|l|l|}
\hline & \multicolumn{2}{|l|}{ Direct } & \multicolumn{2}{l|}{ Water bridged } \\
\hline Occupancy (\%) & Tot. & $>20$ & Tot. & $>20$ \\
\hline $\begin{array}{l}\text { Backbone }- \\
\text { side chain }\end{array}$ & 6 & 2 & 7 & 1 \\
\hline Side chains & 7 & 4 & 7 & 5 \\
\hline
\end{tabular}




\section{Figures}

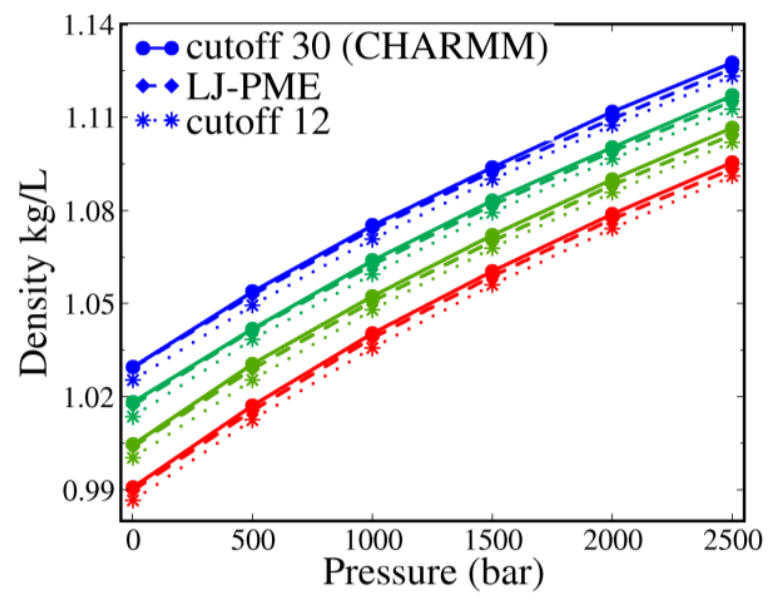

Figure S1. The TIP3P water density with respect to temperature and pressure simulated using different Lennard-Jones computational scheme.

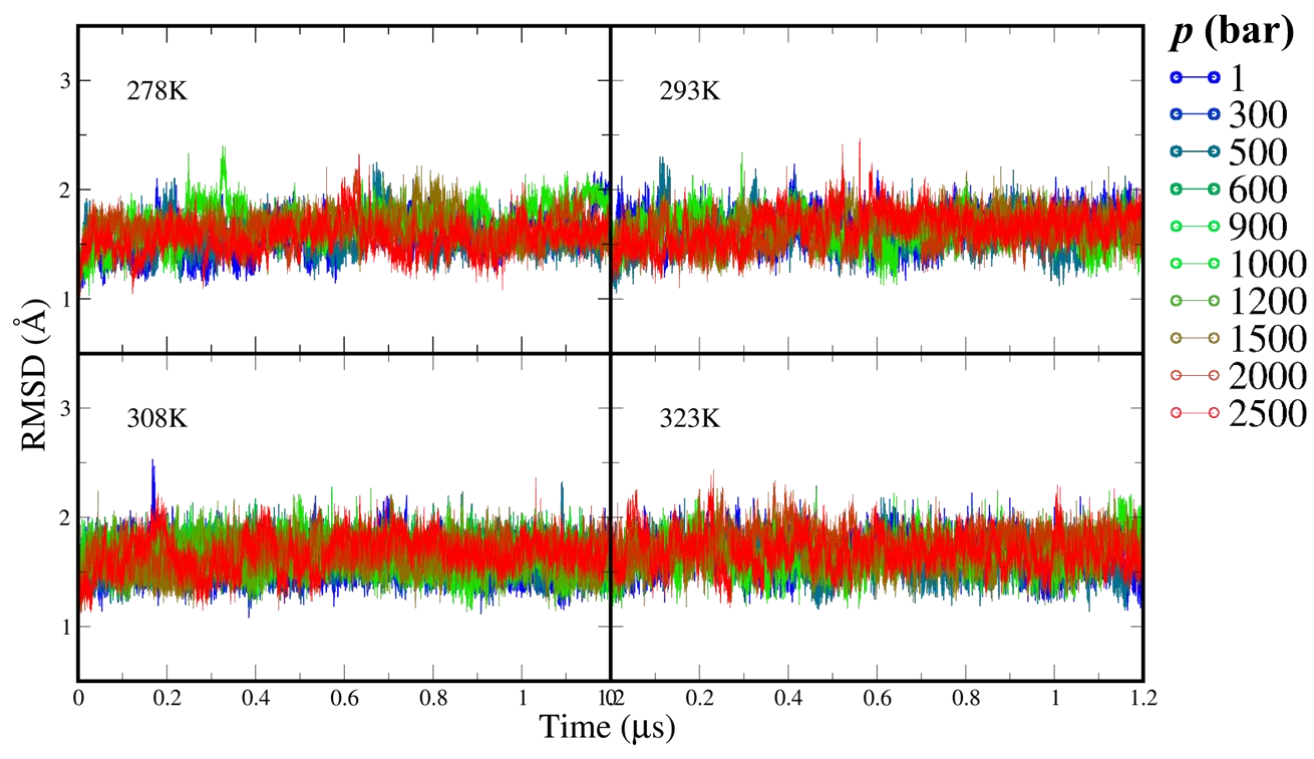

Figure S2. The coordinate RMSD of ubiquitin heavy atoms as the function of the simulation time. 


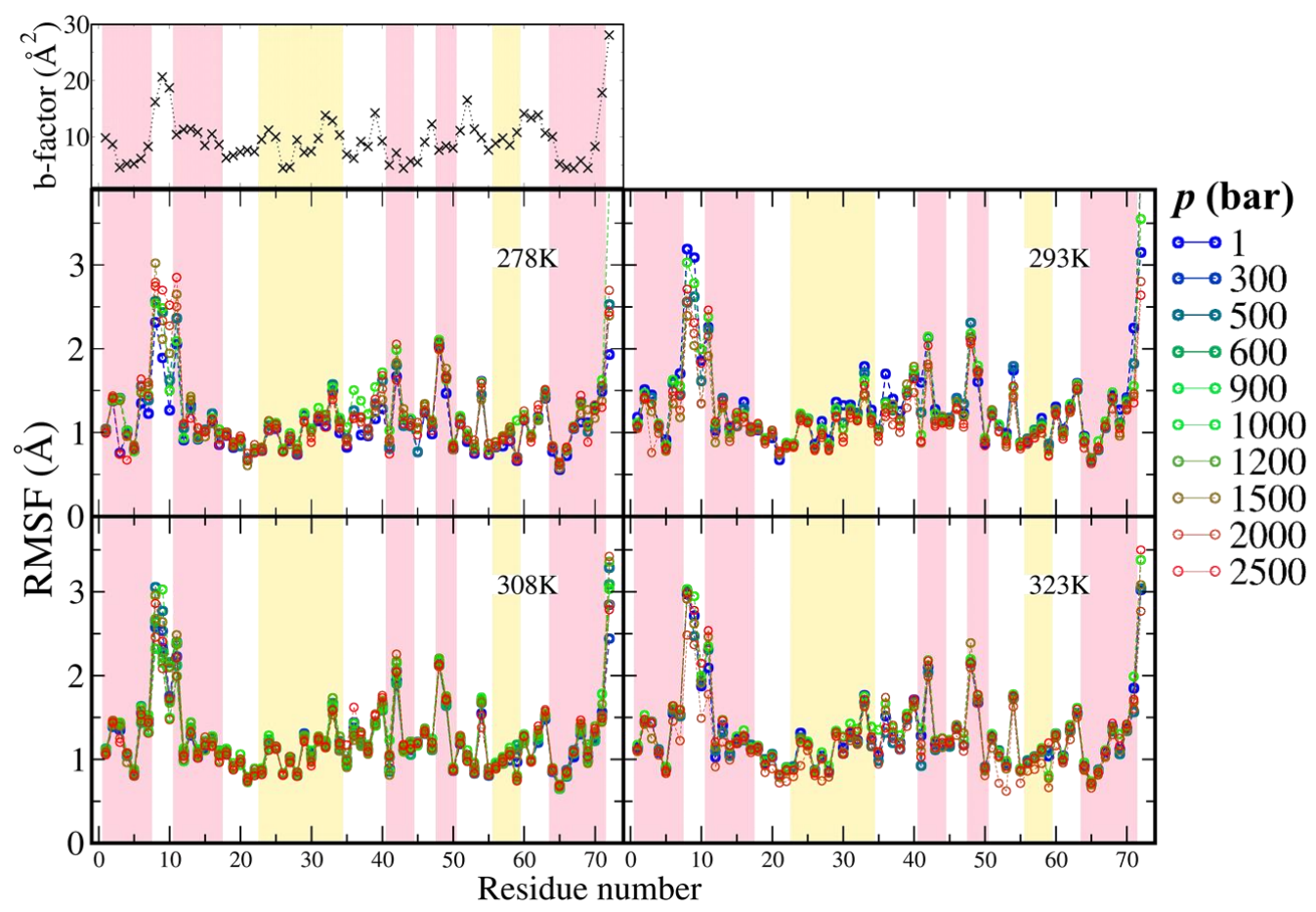

Figure S3. The RMSF of ubiquitin residues $1-72$. The designation of secondary structure domains was colored on panel background, as helices in yellow and $\beta$-strand in pink. The black cross on the top-left indicates the average b-factor per residue of backbone atoms in the crystal structure $1 \mathrm{UBQ}$.

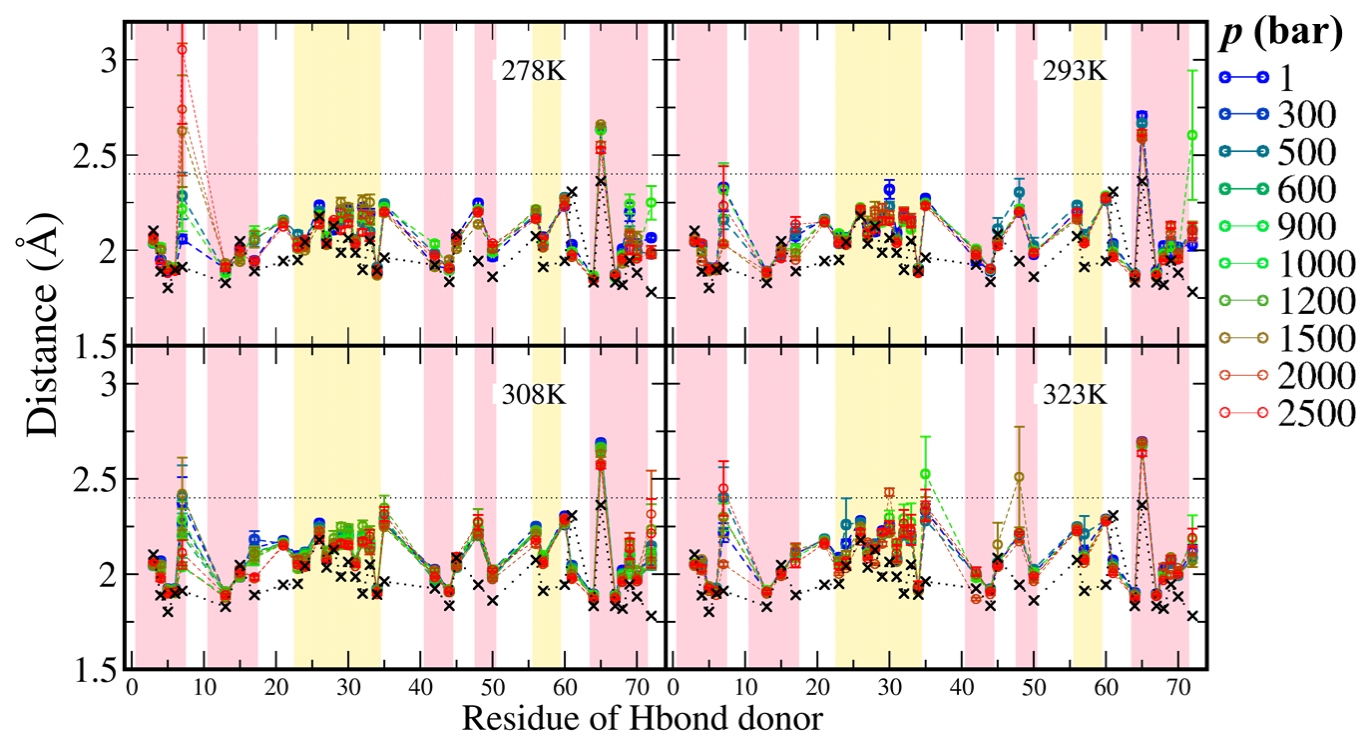

Figure S4. The distance between donor and acceptor of backbone H-bonds in ubiquitin. The hydrogen bonds are indexed by the donor residue. The background is colored as the secondary structure domains, i.e. yellow for helices and pink for sheets. The black cross indicates the HN $\cdots \mathrm{O}$ distance in the crystal structure 1UBQ (hydrogen atoms were added according to $\mathrm{C} 36 \mathrm{~m}$ parameters). The error bars show the averaged block standard deviation. The dotted baselines indicate $2.4 \AA$, under which the interaction is considered to be a strong hydrogen bond. 

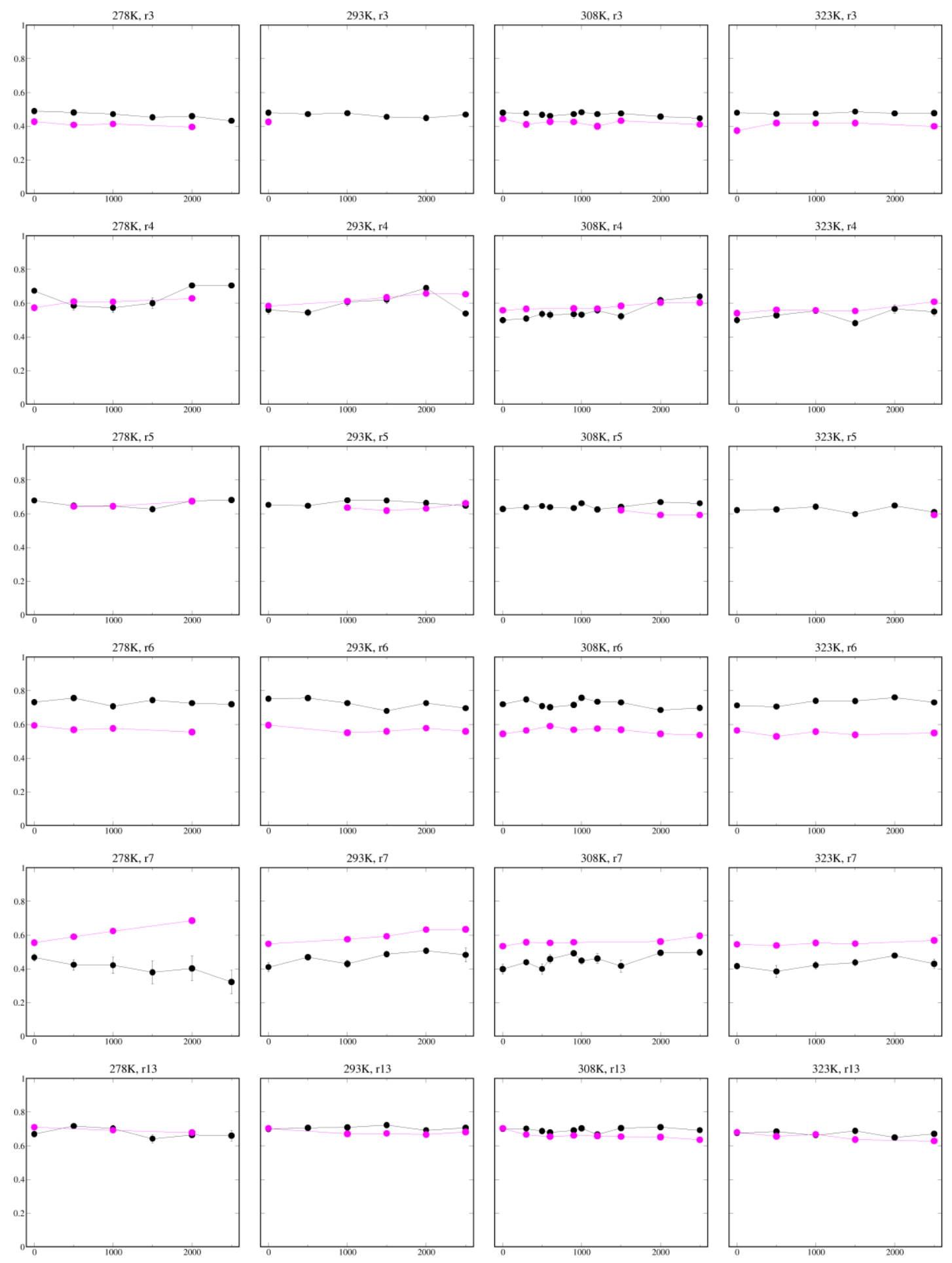

293K, r13
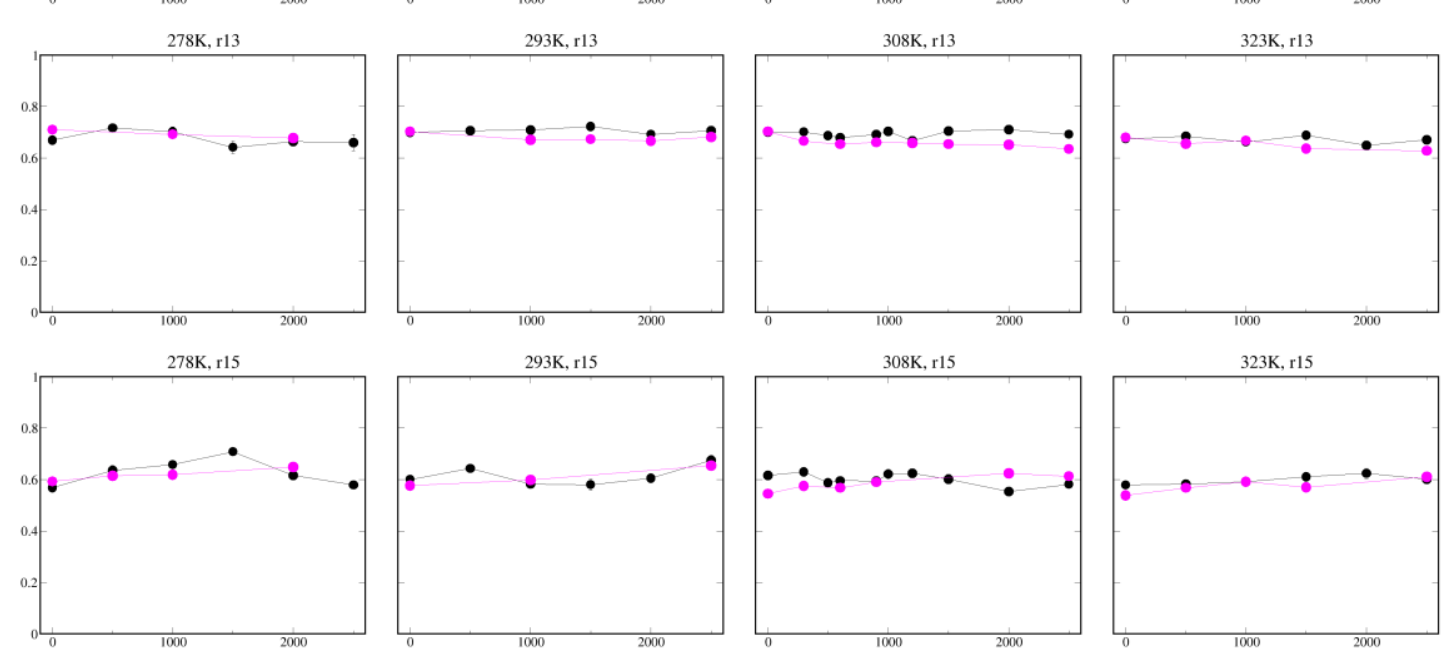

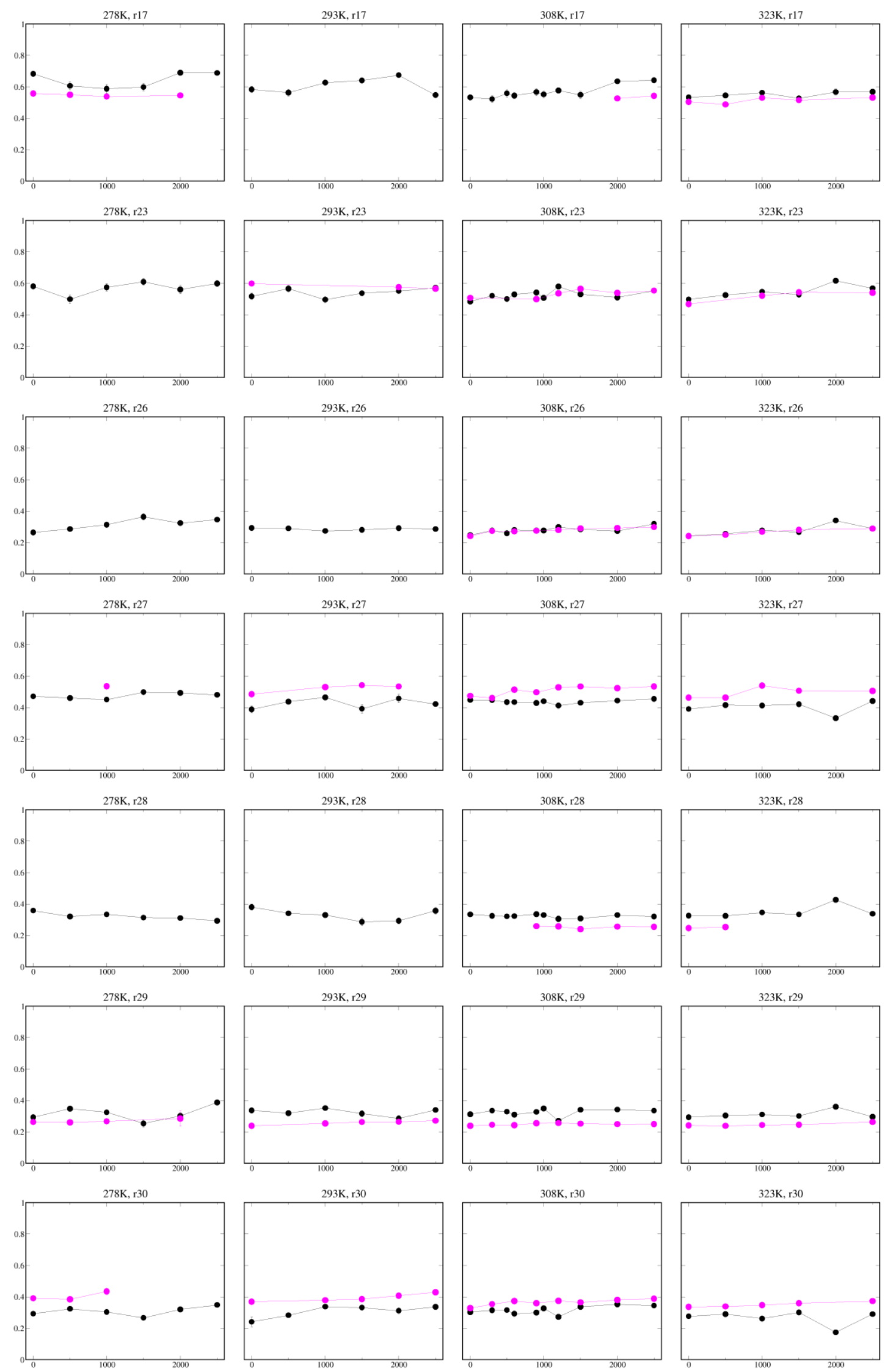

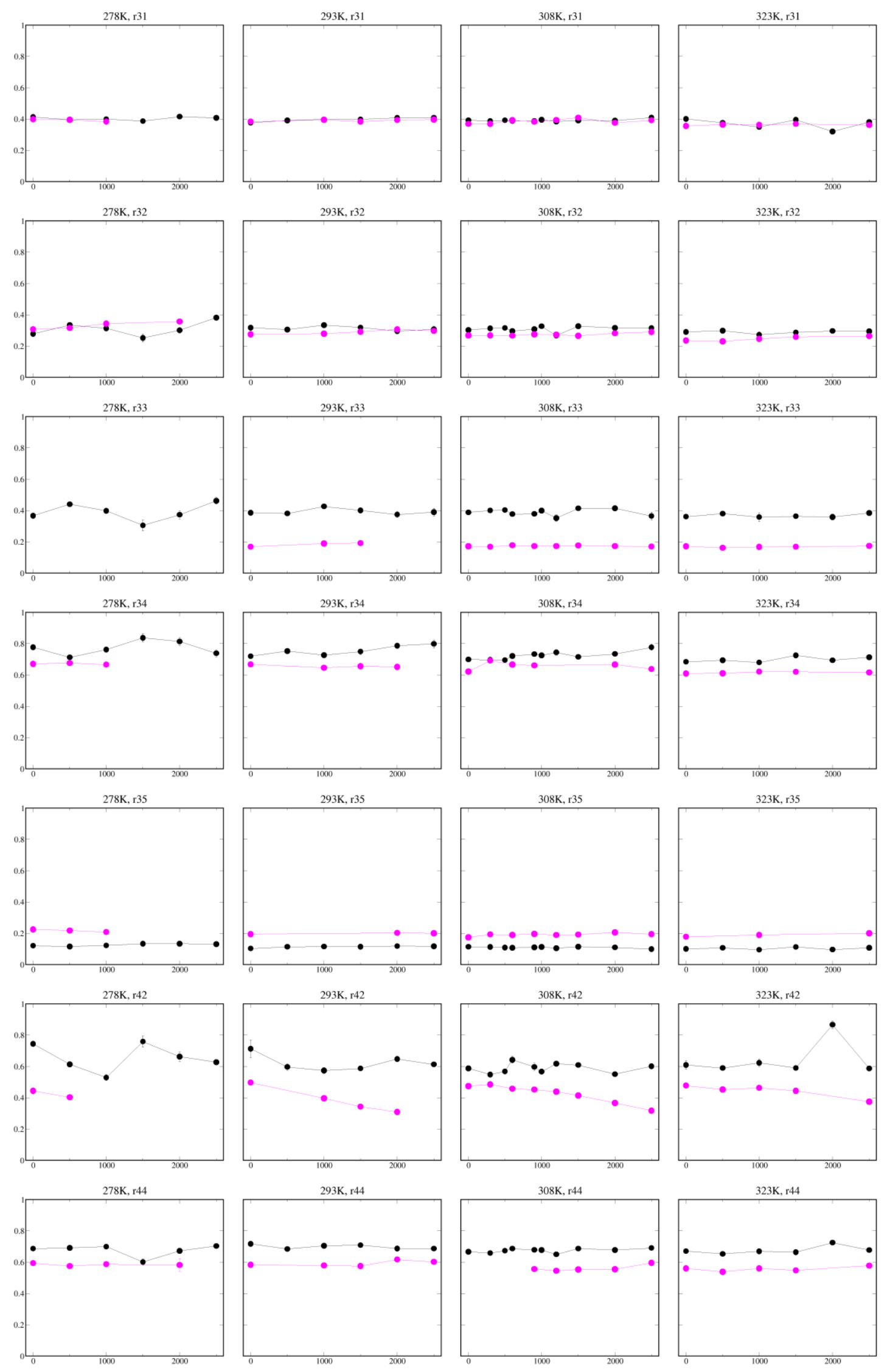

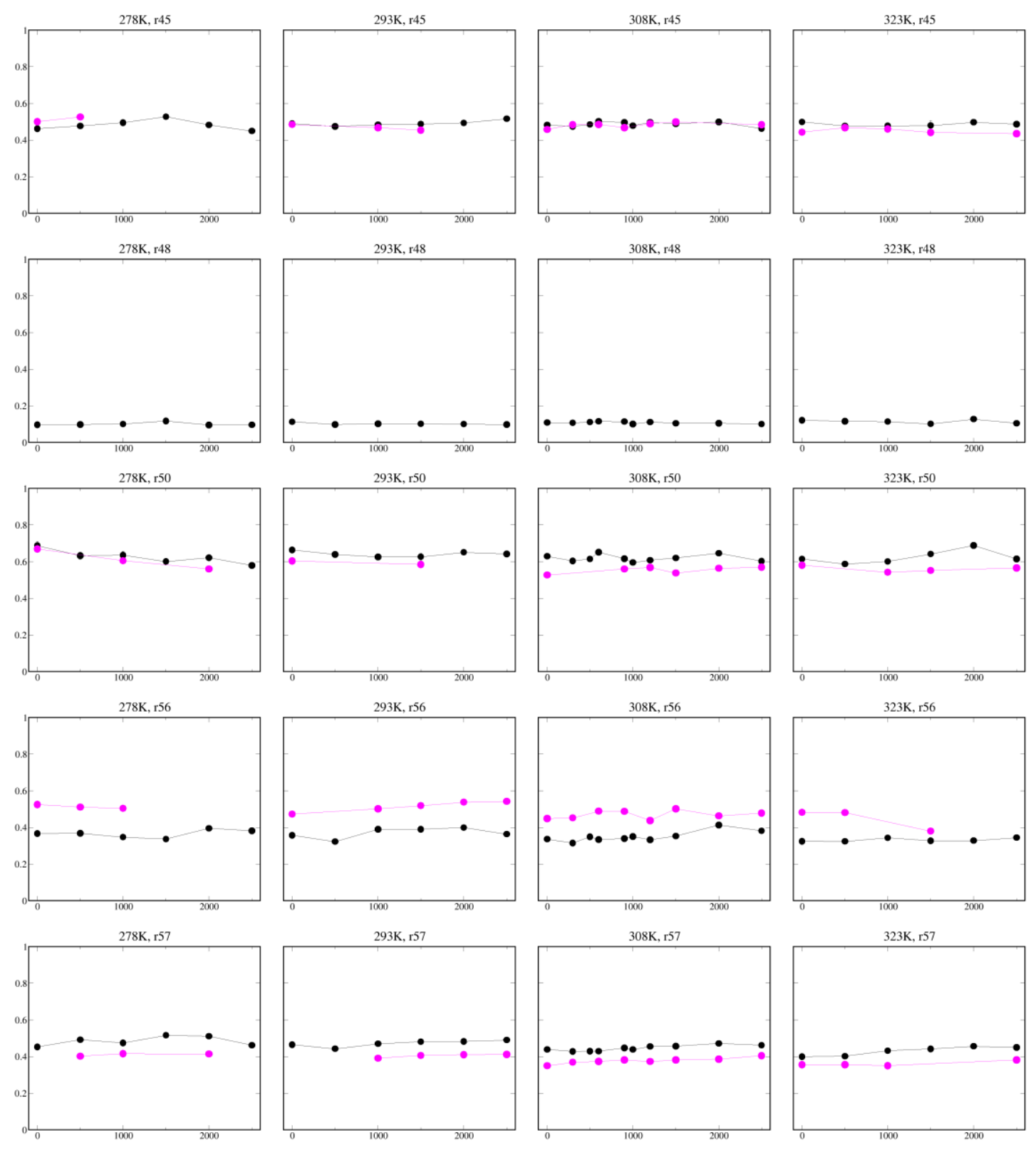

293K, r57
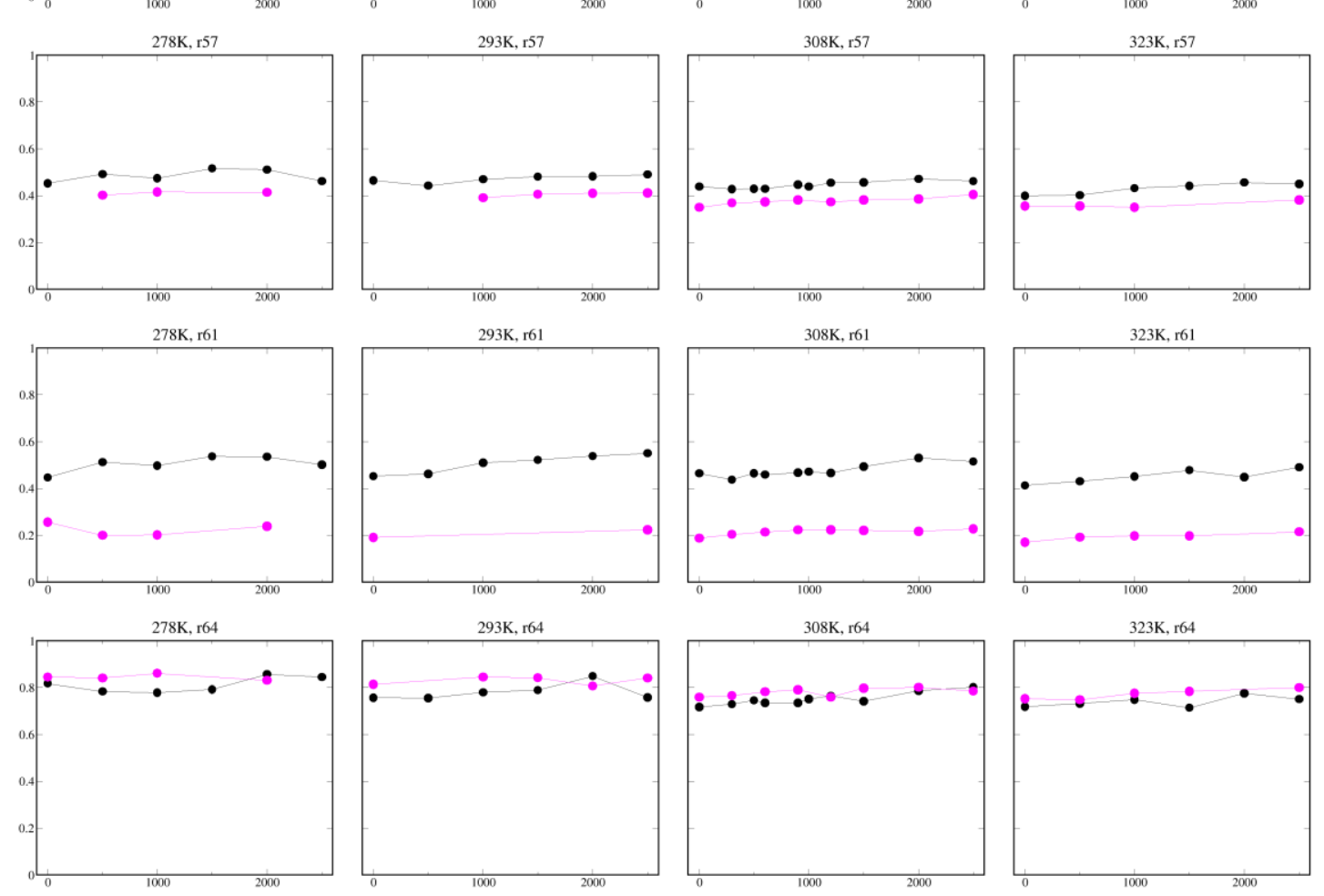

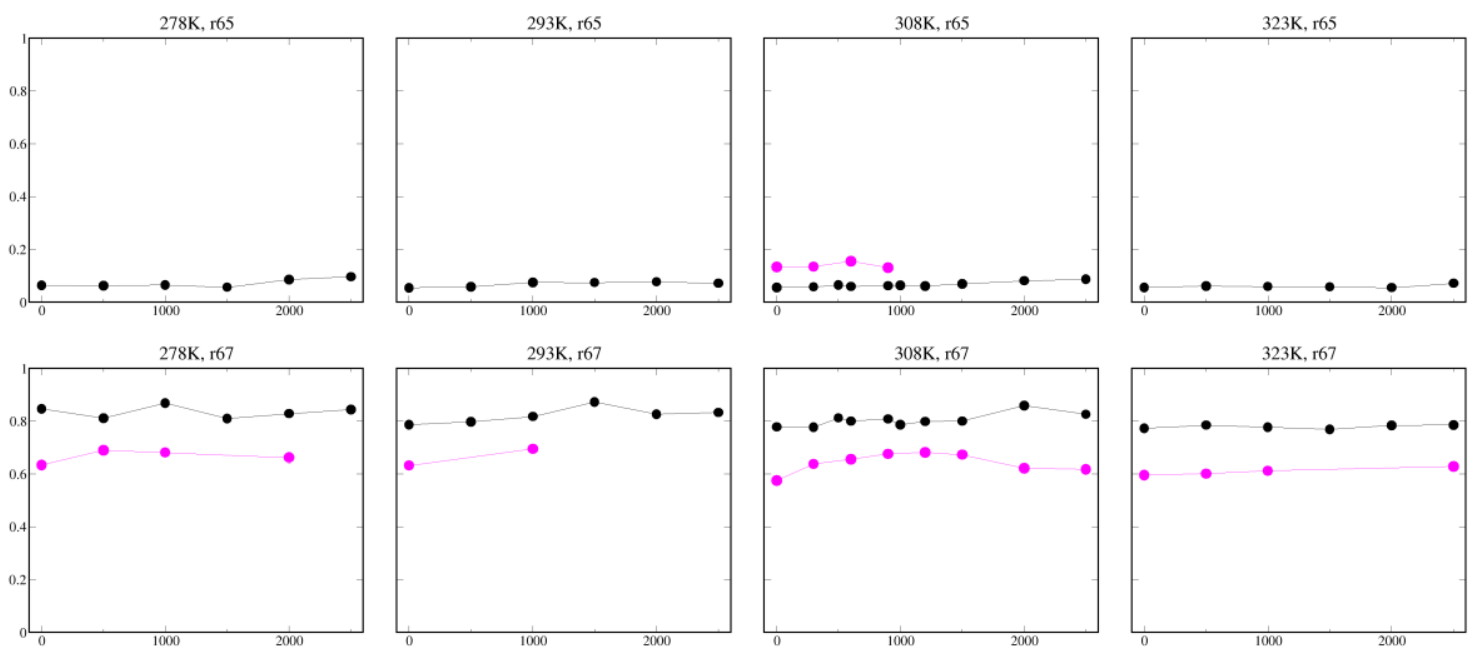

293K, r67

308K, r67

$323 \mathrm{~K}, \mathrm{r} 67$
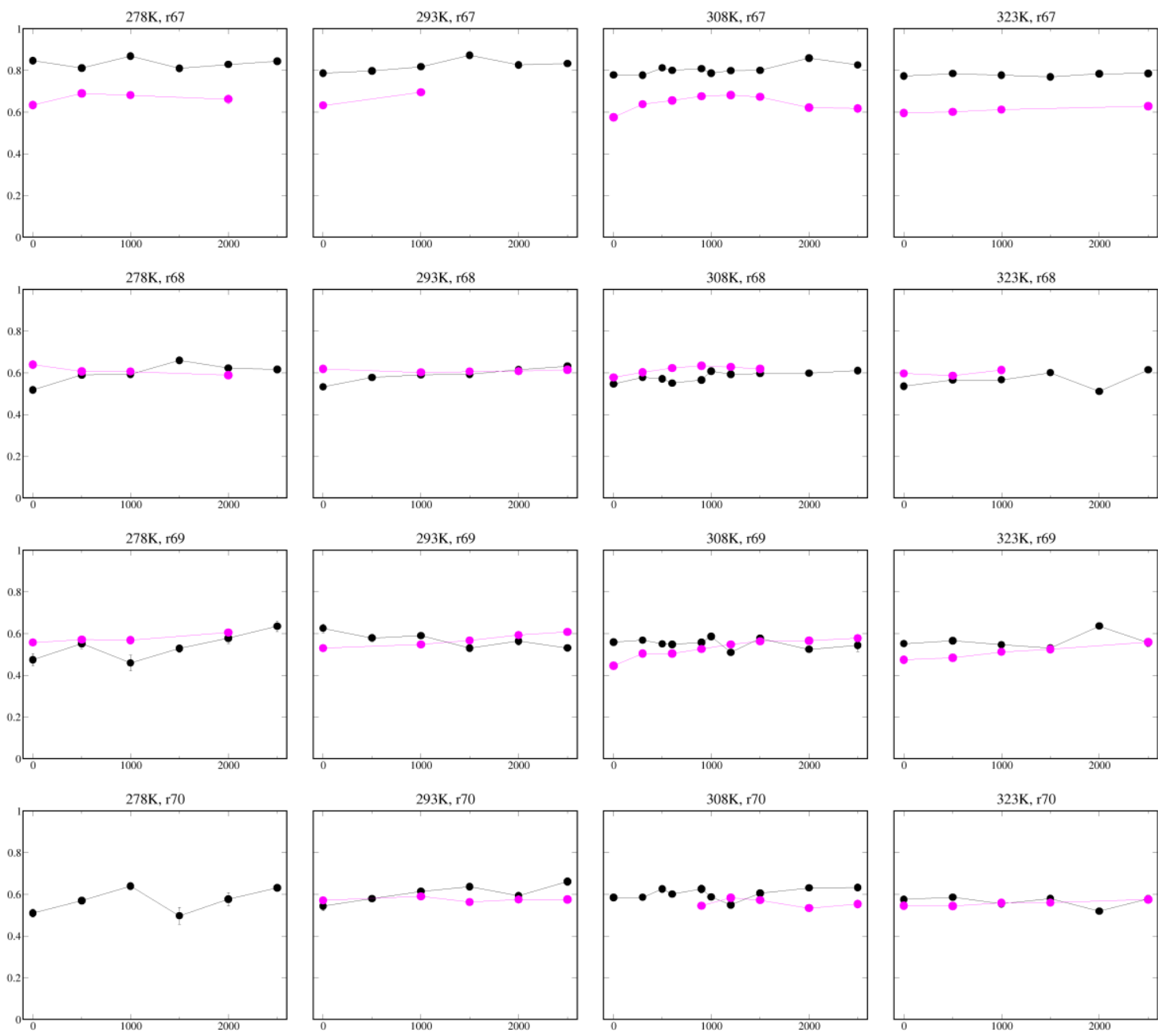

Figure S5. The comparison of residue ${ }^{\mathrm{h} 3} \boldsymbol{J}_{\mathrm{NC}}$ ' with respect to pressure. The calculations are in black and experimental ${ }^{\mathrm{h} 3} J_{\mathrm{NC}}$ data ${ }^{29}$ are in magenta. Each panel is titled by temperature and donor residue identifier. The error bars of calculated data show the averaged block standard deviation. 
hydrophobic_sum_278

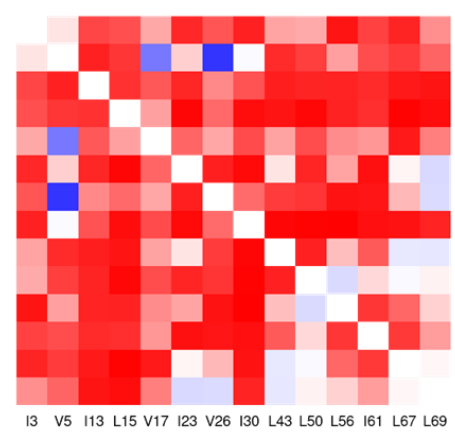

hydrophobic_sum_293

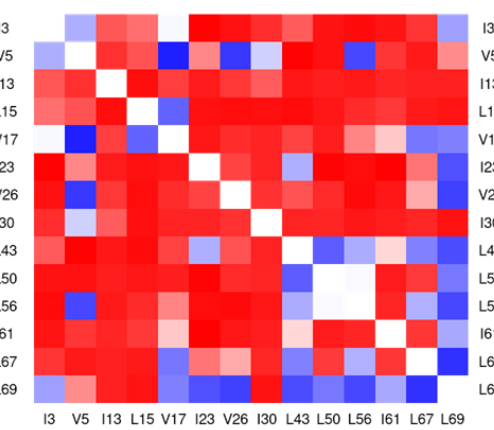

hydrophobic_sum_323

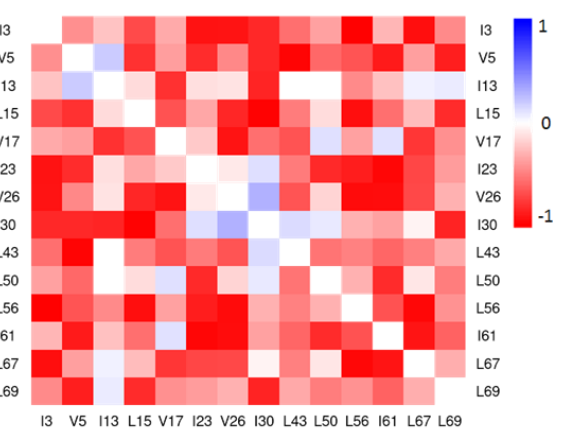

Figure S6. Pearson correlation coefficients between hydrophobic distances and pressure. The pairwise distances were measured between the mass centers of 14 hydrophobic side-chains in the pocket at temperature $278 \mathrm{~K}$ (left), $298 \mathrm{~K}$ (middle) and $323 \mathrm{~K}$ (right). From $\rho=1$ (blue) to $\rho=-1$ (red), the distances correlate with pressure from positively to negatively. 